\title{
The CHY-Type Zinc Finger Protein FgChy1 Regulates Polarized Growth, Pathogenicity, and Microtubule Assembly in Fusarium graminearum
}

\author{
Shulin Cao, ${ }^{1}$ Wei Li, ${ }^{1}$ Chaohui Li, ${ }^{1}$ Guanghui Wang, ${ }^{2}$ Wenqiang Jiang, ${ }^{1,3}$ Haiyan Sun, ${ }^{1}$ Yuanyu Deng, ${ }^{1}$ \\ and Huaigu Chen ${ }^{1,4,+}$ \\ ${ }^{1}$ Institute of Plant Protection, Jiangsu Academy of Agricultural Sciences, Nanjing 210014, Jiangsu, China \\ ${ }^{2}$ State Key Laboratory of Crop Stress Biology for Arid Areas, College of Plant Protection, Northwest A\&F University, Yangling \\ 712100, Shaanxi, China \\ ${ }^{3}$ Hubei Collaborative Innovation Center for Grain Industry, Yangtze University, Jingzhou 434025, Hubei, China \\ ${ }^{4}$ Jiangsu Co-Innovation Center for Modern Production Technology of Grain Crops, Yangzhou University, Yangzhou 225009, \\ Jiangsu, China
}

Accepted 27 December 2020.

\begin{abstract}
Microtubules (MTs), as transport tracks, play important roles in hyphal-tip growth in filamentous fungi, but MT-associated proteins involved in polarized growth remain unknown. Here, we found that one novel zinc finger protein, FgChy1, is required for MT morphology and polarized growth in Fusarium graminearum. The Fgchy1 mutant presented curved and directionless growth of hyphae. Importantly, the conidia and germ tubes of the Fgchy1 mutant exhibited badly damaged and less-organized beta-tubulin cytoskeletons. Compared with the wild type, the Fgchy1 mutant lost the ability to maintain polarity and was also more sensitive to the anti-MT drugs carbendazim and nocodazole, likely due to the impaired MT cytoskeleton. Indeed, the hyphae of the wild type treated with nocodazole exhibited a morphology consistent with that of the Fgchy1 mutant. Interestingly, the disruption of FgChy1 resulted in the off-center localization of actin patches and the polarity-related polarisome protein FgSpa2 from the hyphal-tip axis. A similar defect in FgSpa2 localization was also observed in the nocodazole-treated wild-type strain. In addition, FgChy1 is also required for conidiogenesis, septation, sexual reproduction, pathogenicity, and deoxynivalenol production. Overall, this study provides the first demonstrations of the functions of the novel zinc finger protein FgChy1 in polarized growth, development, and virulence in filamentous fungi.
\end{abstract}

Keywords: beta-tubulin, CHY-type zinc finger, polarity growth, virulence, wheat head blight

${ }^{\dagger}$ Corresponding author: H. Chen; huaigu@ jaas.ac.cn

Funding: This work was supported by the National Key R\&D Program of China (grant number 2018YFD0200500/2018YFD0200505), the Special Fund for China Agricultural Research System (CARS-3) and the Jiangsu Key R\&D Plan (BE2019377).

*The $\boldsymbol{e}$-Xtra logo stands for "electronic extra" and indicates there is supplementary material published online.

The author(s) declare no conflict of interest.

(c) (1) () $\odot$ Copyright $\odot 2021$ The Author(s). This is an open access article (C) (1) () () distributed under the CC BY-NC-ND 4.0 International license.
Fusarium head blight (FHB), caused by the ascomycete pathogen Fusarium graminearum complex, is a devastating disease of wheat and barley worldwide. The outbreak of FHB has a substantial impact on grain quality and yield (Goswami and Kistler 2004). Various types of mycotoxins, including deoxynivalenol (DON) produced on $F$. graminearum-infected grains, are potent inhibitors of eukaryotic protein synthesis and pose a serious threat to human and livestock health. DON has also been identified as a virulence factor in $F$. graminearum and participates in the pathogen spread within plants (Seong et al. 2009; Walter et al. 2010). The biosynthetic enzymes required for DON production in Fusarium spp., including the TRI5 cluster, TRI1 cluster, and TRIIO1 loci, have been extensively investigated (Alexander et al. 2009; Seong et al. 2009). Chemical fungicide control remains a major strategy for FHB management due to the lack of highly resistant wheat cultivars. Carbendazim (methyl 2-benzimidazole carbamate [MBC]) and triazole fungicides, particularly tebuconazole, are widely used in FHB management in China and worldwide. However, the long-term application of these fungicides has given rise to MBC-resistant and tebuconazole-resistant $F$. graminearum strains (Liu et al. 2010; Spolti et al. 2014). Moreover, the application of fungicides, including $\mathrm{MBC}$ and triazoles at sublethal concentrations, could trigger DON biosynthesis (Audenaert et al. 2010; Zhang et al. 2009). Therefore, new candidates for the development of lowtoxicity and efficient fungicides for FHB and DON control are urgently needed.

The microtubule (MT) dynamics and structural stability in eukaryotes play a critical role in many cellular activities, including cell signaling, cell division, and cell shape (Yeh et al. 2000). At present, tubulin is an attractive target of many anticancer drugs, anthelminthics, and fungicides (Kiso et al. 2004). Benzimidazole fungicides (e.g., MBC) disrupt the assembly of MTs by specifically targeting beta-tubulin in Aspergillus nidulans and F. graminearum (Kiso et al. 2004; Qiu et al. 2011). In addition, many anticancer drugs (e.g., vinca alkaloids and taxanes) (Hwang et al. 2013; Ojima et al. 2014) and herbicides (e.g., trifluralin) (Chu et al. 2018) target MTs by suppressing dynamic instability, which ultimately leads to mitotic arrest and cell death induced by colchicine. Therefore, understanding the molecular process of MT-associated factors in detail might lead to the identification of targets for the development of new and safe antifungal drugs. 
It is worth mentioning that MTs, as one of the core factors regulating polarized growth in filamentous fungi, are involved in hyphal directional growth and the maintenance of polarity (Riquelme 2013; Taheri-Talesh et al. 2008). Polarized growth is an intrinsic feature of filamentous fungi and determines the shape, branch, and growth direction (Riquelme 2013). The dominant growth form of filamentous fungi is involved in various biological processes, such as exocytosis, endocytosis, pathogenicity, sexual reproduction, and even signal recognition (Arkowitz 2013; Riquelme 2013). MTs, as tracks, transport secretory vesicles from the cell body to the subapical area and then communicate with the hyphal apex via actin cables (Upadhyay and Shaw 2008). Many MT plus-end tracking proteins that regulate MT dynamic instability play important roles in the maintenance of cell polarity. For instance, EB1 family protein EB1, which is involved in MT modulation, is essential for tip growth in various fungi (Liu et al. 2017). MT polymerases (e.g., Alp14 in fission yeast and AlpA in A. nidulans) involved in the organization of MT dynamics are responsible for hyphal directional growth and polarity maintenance (Takeshita et al. 2013). These findings are clearly attributed to the critical roles of MTs in polarity maintenance and rapid hyphal growth in fungi (Horio and Oakley 2005; Zeng et al. 2014). However, the mechanism of polarized growth regulated by MTs and the MT-associated proteins involved in polarized growth remain unknown.

In this study, we identified FgChy1, a novel CHY-type zinc finger (Zf-CHY) protein involved in MT morphology and pathogenicity in $F$. graminearum. The $\mathrm{Zf}-\mathrm{CHY}$ domain with conserved cysteine and histidine residues is found in most eukaryotic species, including fungi, but little is known about its specific functions. Higher eukaryotes contain a large number of proteins with the Zf-CHY domain, which often coexist with other zinc finger structures (e.g., RING) (Cui et al. 2015; Leng et al. 2003). However, unlike the results observed in higher eukaryotes, only one protein in Saccharomyces cerevisiae, Hot13, harbors the Zf-CHY domain (Curran et al. 2004), and this protein only contains a Zf-CHY domains without any other domains. Hot13 is located in the mitochondrial intermembrane and is needed for the efficient assembly of small translocase of inner membrane (Tim) proteins into complexes (Curran et al. 2004). Orthologs of Hot13 are only conserved in microorganisms such as fungi and oomycetes, although the $\mathrm{Zf}-\mathrm{CHY}$ domain exists in many species ranging from lower to higher organisms. The function of proteins containing only the $\mathrm{Zf}$ CHY domain in microbes other than $S$. cerevisiae is completely unknown.

In this study, three Zf-CHY domain-containing proteins, namely, FgChy1 (Hot13 homology), FgChy2, and FgChy3, were identified in the $F$. graminearum genome. FgChyl was studied in detail; its orthologs are present in fungi and oomycetes such as Phytophthora infestans but absent in bacteria, plants, and animals. We further characterized its functions in vegetative growth, conidiation, sexual reproduction, and pathogenesis in F. graminearum. The Fgchyl mutant exhibited highly curved hyphal growth and severely decreased pathogenicity in $F$. graminearum. This mutant also produced bent conidia and showed defects in septum formation, infectious structure formation, and sexual reproduction. In addition, the bent conidia and wavy hyphae produced by the Fgchyl mutant prompted us to explore the function of $\mathrm{FgCHYl}$ in regulating the polarized growth of $F$. graminearum. We found that deletion of $\mathrm{FgCHY1}$ affected cytoplasmic MT assembly and was specifically associated with anti-MT drug sensitivity. The Fgchyl mutant also showed defects in the localization of FgSpa2 and F-actin at the hyphal apex. Subsequently, we revealed that FgChy1 controls the location of the polarisome protein
FgSpa2 on the hyphal apex by regulating the assembly of betatubulin and thus guides the direction of mycelial growth in $F$. graminearum, and the function of FgChy1 orthologs in regulating polarity maintenance might be conserved in other filamentous fungi.

\section{RESULTS}

\section{Identification of CHY zinc finger domain-containing proteins in $F$. graminearum.}

Zf-CHY domain-containing proteins encoded by FGSG_07281 (designated $\mathrm{FgCHYl}$ ) were first identified through a BLASTP search of the EnsemblFungi $F$. graminearum genome database, with Hot13 of $S$. cerevisiae as the query. In addition to $\mathrm{FgCHYl}$, two other Zf-CHY-containing genes, FGSG_09462 (designated FgCHY2) and FGSG_01536 (designated $F g C H Y 3$ ), were identified. Subsequent alignments showed that the amino acid sequences of the three $\mathrm{Zf}-\mathrm{CHY}$-containing proteins exhibited great variance, only conserved in the cysteine residues in the $\mathrm{Zf}-\mathrm{CHY}$ domain (Supplementary Fig. S1A). FgChy1 was predicted to encode a short protein (127 amino acids) and harbors the Zf-CHY domain in its N terminus (Supplementary Fig. S1B). In contrast, $\mathrm{FgCHY} 2$ was predicted to encode a longer protein with 686 amino acids and possessed the Zf-CHY domain in its $\mathrm{C}$ terminus (Supplementary Fig. S1B). No other domains other than Zf-CHY were found in either FgChy1 or FgChy2, whereas Zf-CHY coupled with the ring finger (RCHY domain) was localized in the $\mathrm{C}$ terminus of FgChy3 (Supplementary Fig. S1B). These results suggest that the three $\mathrm{Zf}-\mathrm{CHY}$ proteins assume diverse functions in F. graminearum.

Furthermore, sequence alignment and phylogenetic analyses revealed that FgChy1 homologs are present in fungi (including members of divisions Ascomycota, Basidiomycota, Mucoromycota, Zoopagomycota, and Chytridiomycota) and oomycetes (Phytophthora infestans) but are absent in bacteria, plants, and animals (Supplementary Fig. S1C and D). FgChy2 is present in fungi and oomycetes, excluding members of order Saccharomycetales and class Basidiomycetes (Supplementary Fig. S1E). However, FgChy3 is conserved from lower eukaryotes to higher plants and animals (Supplementary Fig. S1F), which suggests the divergence of CHY-type proteins during evolution. FgChy1 displays low homology with yeast Hot 13 , with only approximately $30 \%$ identity in the Zf-CHY domain and great variance outside this domain. In contrast, FgChy1 orthologs are highly conserved in filamentous fungi. Moreover, FgChy1 shows greater sequence similarity to its orthologs in Schizosaccharomyces pombe (with 54\% identity) and even $P$. infestans (with $53 \%$ identity) than to its ortholog in $S$. cerevisiae (Supplementary Fig. S1C). Presumably, FgChy1 homologs of filamentous microbes or even Schizosaccharomyces pombe might share similar functions that are different from those of orthologs in Saccharomycetales species.

\section{Generation and characterization}

\section{of the predicted $\mathrm{Zf}$-CHY gene-deletion mutants.}

We generated Fgchy1, Fgchy2, and Fgchy3 mutants using the split-marker approach (Catlett et al. 2003). The hygromycinresistant transformants were screened by PCR with four primer pairs, as previously described (Wang et al. 2011) (Supplementary Table S1; Supplementary Fig. S2). At least two deletion mutants were obtained for $\mathrm{FgCHY1}, \mathrm{FgCHY}$, and $\mathrm{FgCHY}$. Fgchy2 and $F g c h y 3$ showed unrecognizable changes in colony morphology on complete medium (CM) for 3 days, whereas Fgchyl grew markedly more slowly than the wild-type strain (Fig. 1A). The wheat infection assay indicated that the Fgchyl mutant significantly decreased virulence, and its typical symptoms on 
the inoculated kernels barely spread to neighboring spikelets at 14 days postinoculation (dpi) (Fig. 1B). Under the same conditions, Fgchy 2 and Fgchy3 mutants displayed a similar infection ability to the wild-type strain. Therefore, $\mathrm{FgCHY1}$ plays an important role in the vegetative growth and pathogenicity of $F$. graminearum and was thus further characterized in this study.

Complementation and subcellular localization of FgChy1.

The $\mathrm{FgCHYl-GFP}$ (green fluorescent protein) fusion construct that expressed the wild-type $F g C H Y 1$ allele was generated and transformed into the Fgchyl mutant to confirm that the phenotypic defects observed in Fgchyl were due to the deletion of $F g C H Y 1$. The GFP label was used to observe the localization of FgChy1. The resulting complementary strain (named CFgchyl) showed normal vegetative growth, reproduction, and plant infection (Figs. 2, 3, 4, 5 and 6).

In contrast to Hot13, a Zf-CHY protein in S. cerevisiae localized to the mitochondria (Curran et al. 2004), FgChy1 was observed, in whole cells except vacuole compartments, through GFP labeling and 4',6-diamidino-2-phenylindole (DAPI) staining examination (Fig. 1C and D), and the FgChy1 localization has no obvious difference in conidia and growing hyphae. The different distribution features of the Zf-CHY protein might be responsible for the different functional pathways in $F$. graminearum and $S$. cerevisiae.
FgCHY1 plays a critical role in mycelial growth and conidiogenesis.

Compared with the wild type, the growth rate of the Fgchyl mutant was reduced by approximately $70.4 \%$ after incubation on potato dextrose agar (PDA) plates for 4 days (Fig. 2A; Table 1). Colonies formed by the Fgchyl mutant had denser and shorter mycelia clinging to the plate compared with those of PH-1 and CFgchyl as well as orderly and exuberant aerial hyphae (Fig. 2A).

We examined the conidial morphology of the wild-type, Fgchyl, and CFgchyl strains in carboxymethylcellulose (CMC) medium. As shown in Figure 3B, the conidia of the Fgchyl mutant showed different degrees of bending. Compared with the typical slender canoe-shaped morphology of $F$. graminearum conidia, one end of $52 \%$ of the mutant conidia was elongated and strongly curved, forming hook-like structures (Fig. 2B and C). In comparison with the wild type, Fgchyl conidiation was reduced by approximately $38 \%$, which was not proportional to its significant reduction in growth rate (Table 1). Microscopic examination revealed that the phialides produced by Fgchyl were approximately threefold greater in number than those produced by $\mathrm{PH}-1$ and the complementary strain and hook-like structures formed at the tip compartments of the conidia (Fig. 2D). These results suggest that the Fgchyl mutant shows defects in mycelial growth and conidial morphology but facilitates conidiophore development.
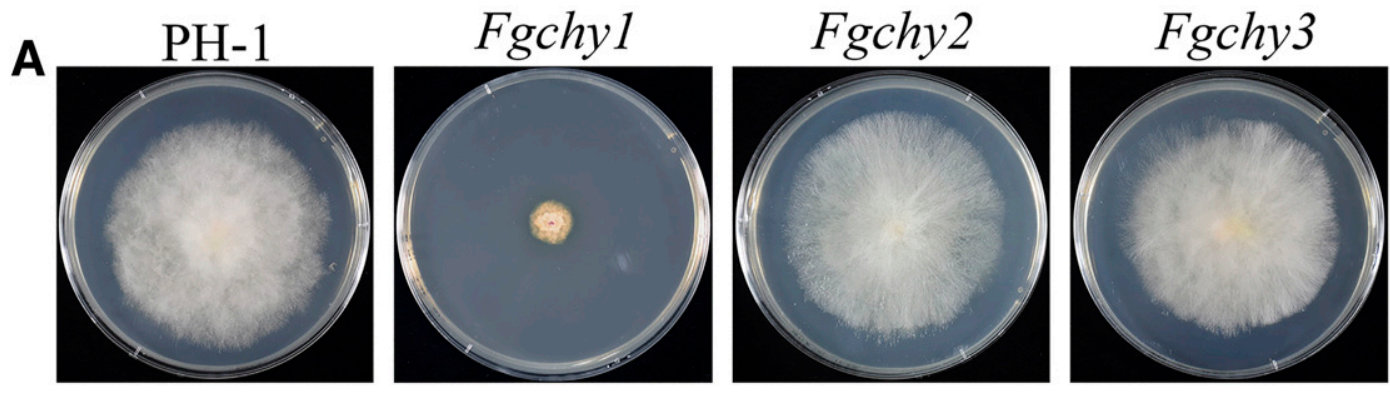

B

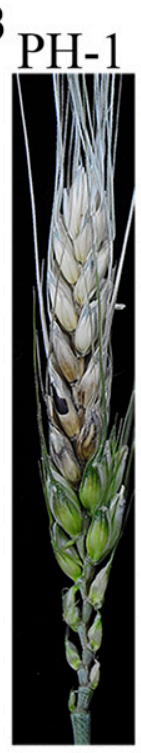

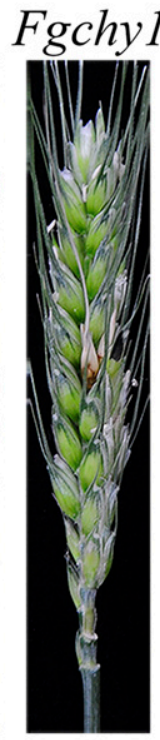
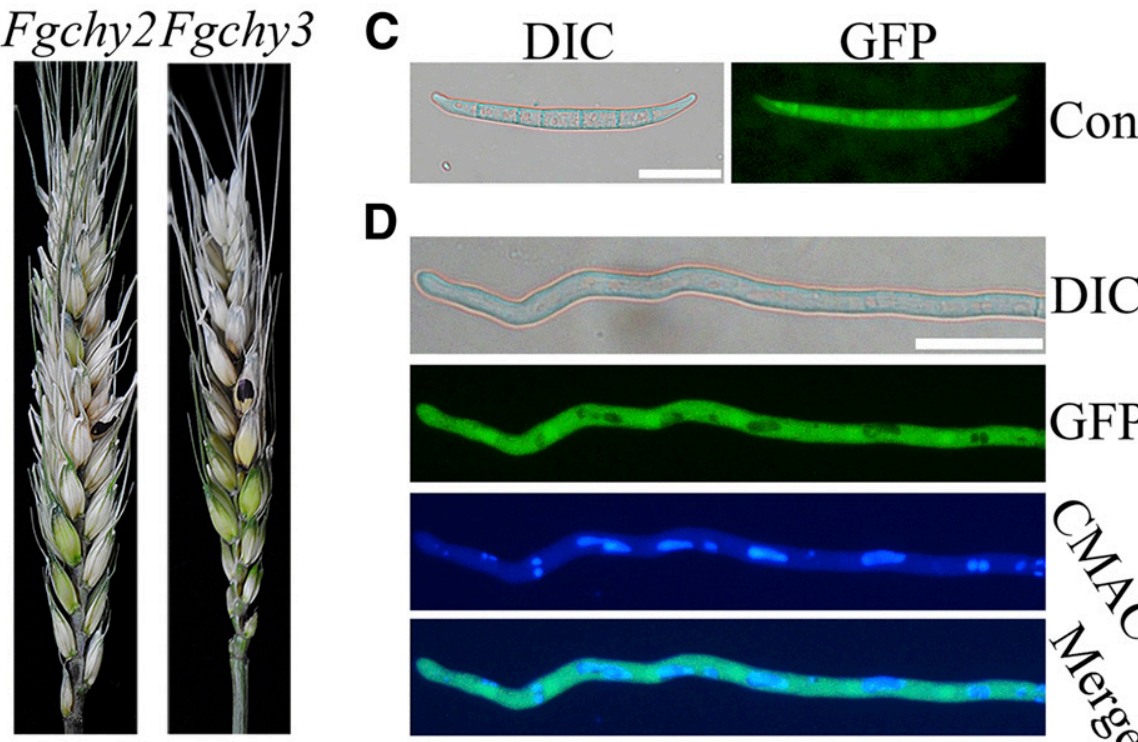

D
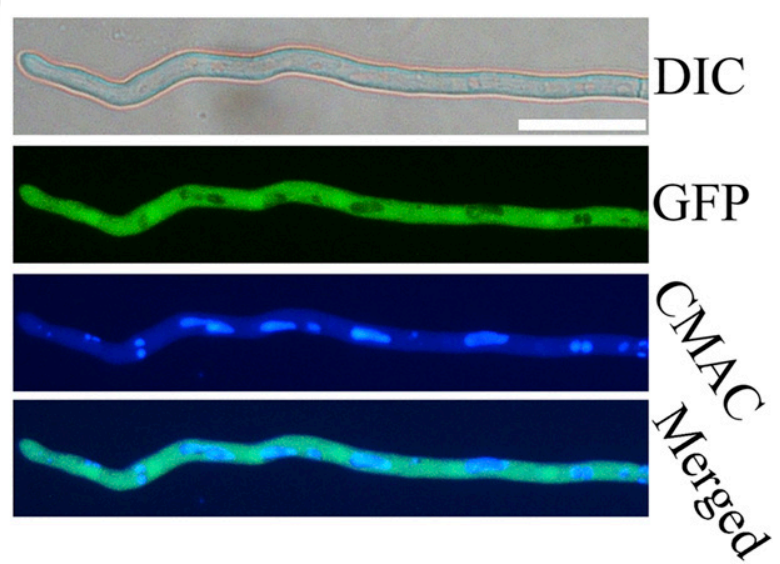

Fig. 1. Character of three CHY zinc finger (Zf-CHY) proteins in Fusarium graminearum. A, Hyphal growth on complete medium of the wild-type strain PH-1 and mutants Fgchyl, Fgchy2, and Fgchy3. B, Scab disease developed on flowering wheat heads inoculated with PH-1 and each mutant. C, Localization of FgChy1 in conidia (con). DIC = differential interference contrast, GFP = green fluorescent protein. D, Location of FgChy1 in hyphae. CMAC (7-amino-4chloromethylcoumarin) was used to stain the vacuoles. 


\section{FgCHY1 is essential for hyphal unidirectional growth.}

The dense vegetative hyphae and obviously inhibited growth of the Fgchyl mutant encouraged us to examine the colony edge by microscopy. When cultured on one-half CM for $36 \mathrm{~h}$, the Fgchyl mutant produced dense, meandering, and wavylooking hyphae, whereas the wild type and complementary strains produced hyphae with regular edges and growth in an approximately straight line (Fig. 3A). A similar characteristic was also observed after $10 \mathrm{~h}$ of germination in liquid yeast extract peptone dextrose (YEPD). The young hyphae in the wild-type strain exhibited sustained unidirectional growth and normally straight appearances, whereas the Fgchyl hyphae
A

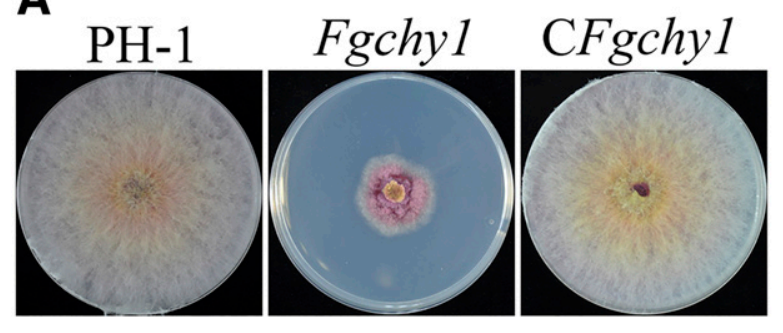

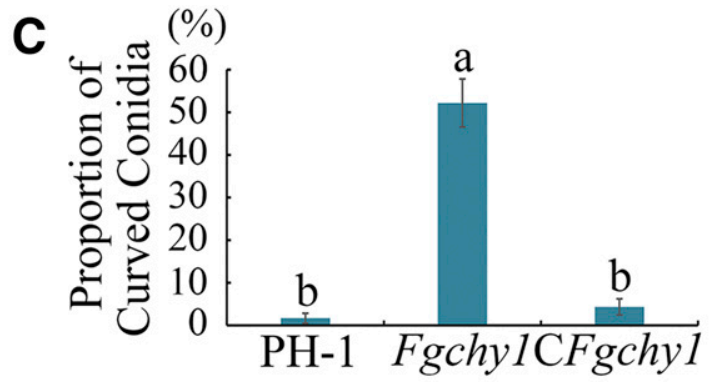

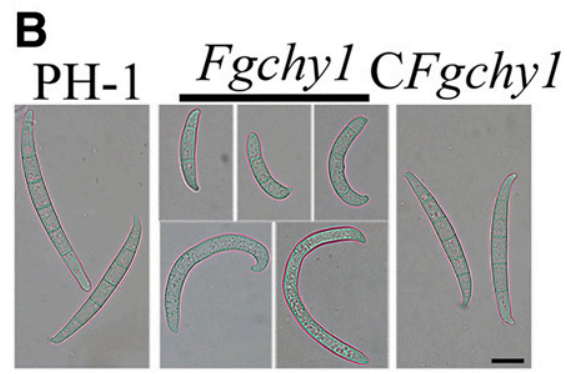

D
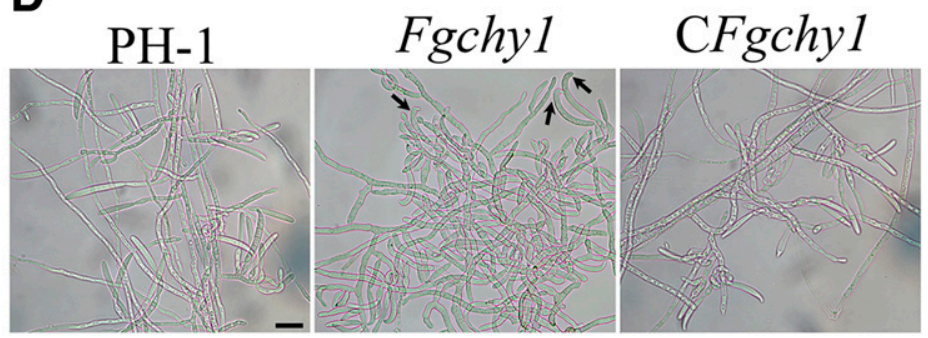

Fig. 2. FgChy1 is required for colony growth and conidial morphology. A, Wild-type strain PH-1, the Fgchyl mutant, and complementary strain CFgchyl cultured on potato dextrose agar medium for 4 days. The growth phenotype of CFgchyl was similar to that of the wild type. B, Conidial morphology of PH-1, the Fgchyl mutant, and CFgchyl. C, Quantification of the percentage of curved conidia in each strain. Means and standard deviations were calculated from three replicates. Different letters indicate significant difference $(P<0.05)$. D, Conidiophore of PH-1, the Fgchyl mutant, and CFgchyl. Black arrows indicate curved tip cells of conidia from the Fgchy1 mutant. Bar $=20 \mu \mathrm{m}$.

A

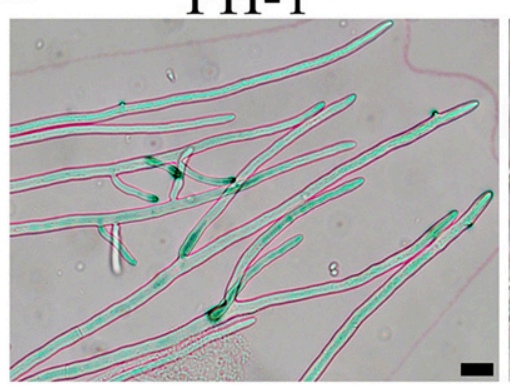

B

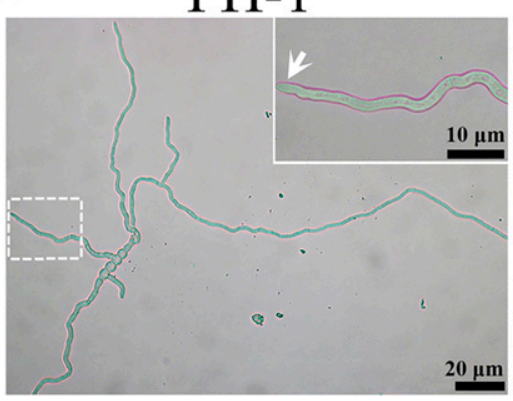

Fgchyl

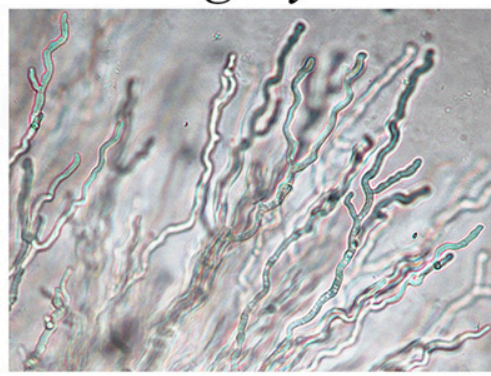

Fgchyl

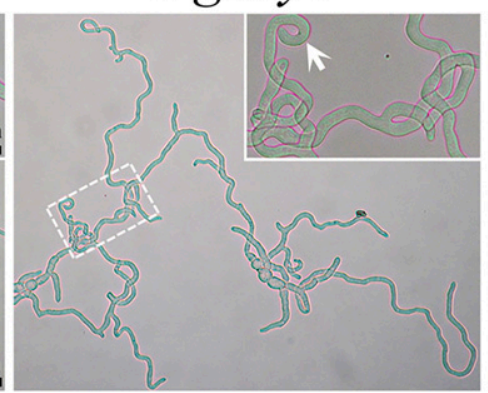

CFgchyl

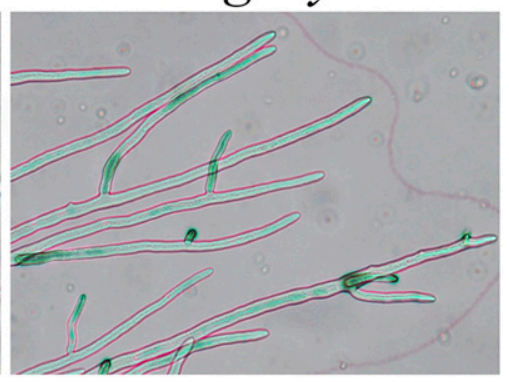

CFgchyl

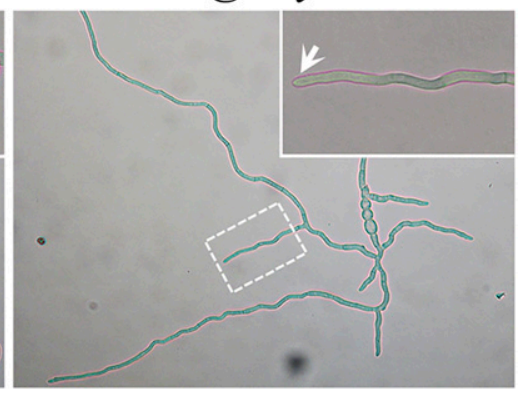

Fig. 3. FgCHY1 is essential for polarity maintenance. A, Colony edge of PH-1, the Fgchyl mutant, and CFgchyl. Bar = 20 $\mu$ m. B, Germlings (after germination for 12 h) of PH-1, the Fgchyl mutant, and CFgchyl in yeast extract peptone dextrose medium. Hyphal tips are marked with arrows. 
showed highly wavy or curved patterns (Fig. 3B). These results indicate that $\mathrm{FgCHYl}$ is important for the maintenance of polarized growth.

\section{Deletion of $\mathrm{FgCHY} 1$ affects septum formation and nuclear distribution.}

We expressed the H1-GFP fusion construct in the Fgchyl mutant and stained it with Calcofluor white $(\mathrm{CFW})$ to determine whether $\mathrm{FgCHYl}$ deletion affects cytokinesis. As shown in Figure $4 \mathrm{~A}$ and $\mathrm{B}$, the majority of conidia produced by PH-1 (84\%) and CFgchyl (80\%) had a normal number of septa (four to seven). In contrast, $66 \%$ of the conidia produced by the Fgchyl mutant had fewer than four septa. The conidia with fewer septa often contained two to three nuclei per cell, which differed from the one nucleus per cell found in normal conidia. More obviously, the septa of the Fgchyl mutant were significantly decreased in vegetative hyphae, and only a few septa formed in complete hyphae (Fig. 4C). These results suggest that $\mathrm{FgCHYl}$ is required for septum formation or cytokinesis.

\section{The Fgchy1 mutant exhibits defects}

in perithecia formation and ascosporogenesis.

The PH-1 and CFgchyl strains produced abundant black mature perithecia with fascicle asci after 14 days of selffertilization (Fig. 5A). Under the same conditions, the Fgchyl mutant formed smaller perithecia (19\% type 2, immature perithecia; $81 \%$ type 3 , proto-perithecia) than the wild type. In addition, the immature perithecia failed to form asci or ascospores (Fig. 5A and B). However, the number of perithecia produced by the Fgchyl mutant was $500 \%$ greater than that obtained with the wild type. These results indicate that $\mathrm{FgCHYI}$ is responsible for sexual development, perithecium maturity, and ascus development.

The Fgchyl mutant was outcrossed with the mat 1-1-1 mutant to confirm the male and female functions of $\mathrm{FgCHYl}$ in sexual development (Cao et al. 2016). Similar to the results obtained with the wild type, normal perithecia and ascospores were produced when Fgchyl was used as the male (Fig. 5C). In contrast, when the Fgchyl mutant was used as the female, the perithecia, similar to those formed by the Fgchyl mutant selfcrosses, were small and sterile (Fig. 5C). Therefore, $\mathrm{FgCHYl}$ is essential for female fertility in $F$. graminearum.

\section{$\mathrm{FgCHY1}$ is important for pathogenicity and DON production.}

At 14 dpi in the infection assay, the average disease index values of the Fgchyl mutant and $\mathrm{PH}-1$ on the flowering wheat heads were 1.0 and 16.6, respectively, which corresponded to an approximately $94 \%$ reduction in virulence (Table 1; Fig. 6A). Consistently, the Fgchyl mutant also caused attenuated virulence on corn silks with only limited brown lesions in the inoculated sites (Fig. 6B).

To further determine the infection process of the Fgchyl mutant in the wheat head, the inoculated lemma were examined by scanning electron microscopy (SEM) at $36 \mathrm{~h}$ postinfection (hpi). In the wild-type strain, direct penetration of epidermal cells via infection hyphae or foot structures was observed at this time, and infection cushions of PH-1 began to form (Fig. 6C).
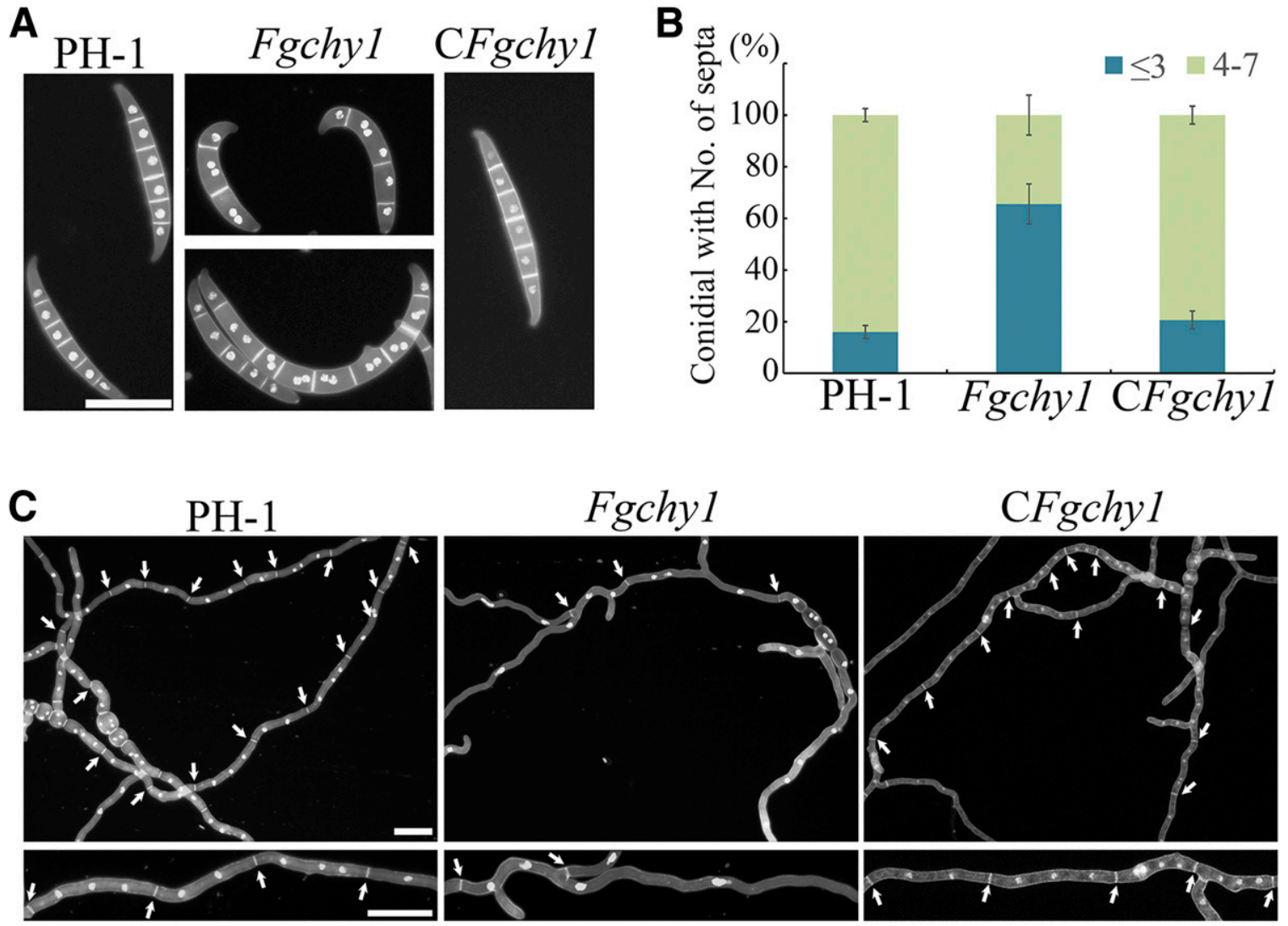

Fig. 4. FgCHY1 is required for septum formation. A, Conidia from transformants of PH-1 and the Fgchyl mutant expressing the H1-GFP (green fluorescent protein) construct (PH-1/H1-GFP and Fgchyl/H1-GFP, respectively) were stained with calcofluor white (CFW) and were examined by light microscope under fluorescein isothiocyanate and UV. Bar $=20 \mu \mathrm{m}$. Conidia of complementary strain CFgchyl were stained with 4',6-diamidino-2-phenylindole (DAPI) and CFW. B, Quantification of the percentage of spores with the number of septa $\leq 3$ and 4 to 7 . At least 300 spores were counted in at least three fields for each strain. Error bars represent standard deviation. C, Hyphae of transformants PH-1 and the Fgchyl mutant were stained with CFW. Hyphae of CFgchyl were stained with DAPI and CFW. The Fgchyl mutant was defective in septum formation. Septa was marked with white arrows. Bar $=20 \mu \mathrm{m}$. 
However, no infection structures but some tortuous hyphae were visible on the surface of the Fgchyl-infected lemma and did not penetrate through the epidermis (Fig. 6C). These results suggest that $\mathrm{FgCHY} 1$ is required for infection structure formation and penetration at the early infection stage.

DON is an important virulence factor in the extension of F. graminearum in the host plant (Seong et al. 2009; Starkey et al. 2007). In the present study, we assayed the production of DON in infectious wheat kernels. The DON concentration of the Fgchyl mutant (approximately $35 \mathrm{ppm}$ ) was lower than those of the wild-type strain (360 ppm) and the complementary strain (308 ppm) (Fig. 7A). Moreover, the expression levels of the DON synthesis genes TRI6, TRI10, TRI12, and TRI101 in the Fgchyl mutant decreased to different degrees relative to those in the PH-1 and CFgchyl strains (Fig. 7B). Previous research has suggested that hyphal bulbous structures at the toxigenic stage are related to DON production (Jiang et al. 2016). When cultured in trichothecene biosynthesis induction medium, bulbous structures were rarely observed in the Fgchyl mutant, and under the same conditions, abundant bulbous structures were observed in the PH-1 and complemented strains (Fig. 7C), which is consistent with decreased DON production. This result suggests that $\mathrm{FgCHYI}$ plays an important role in regulating the production of DON in $F$. graminearum and likely contributes to reduced virulence during infection in wheat.
FgCHY1 is responsible for tip directional growth, probably by regulating the localization and expression of MTs.

MTs are reportedly responsible for maintenance of the directionality of tip growth in A. nidulans (Taheri-Talesh et al. 2008). We first analyzed the expression levels of $F g T U B 1$ and FgTUB2 in wild type and the Fgchyl mutant by quantitative real-time (qRT)-PCR. The expression levels of FgTUB1 and FgTUB2 were lower in the Fgchyl mutant than in the wild-type strain (Fig. 8A). Furthermore, FgTub1-GFP (Wang et al. 2019) and FgTub2-GFP (Wang et al. 2019) were expressed and transformed into PH-1 and Fgchyl mutant to confirm the relationship between FgChy1 and MTs. In the wild type, filaments of FgTub1 and FgTub2 assembled into cytoplasmic MTs parallel to their growth axes and formed mesh networks (Fig. 8B and C). In contrast, in the Fgchyl mutant, the fluorescence intensity of the MTs significantly decreased, and the filaments of FgTub2 were reduced and exhibited disconnection and damage (Fig. 8D). Moreover, hardly any filaments of FgTub1 were detected in the Fgchyl mutant (Fig. 8E). In addition, the location of FgChy1 was not affected when CFgchyl was treated with nocodazole (Noco) (Supplementary Fig. S3B). Thus, Chy1 might serve as an upregulator of MTs and Chy1 is not the target of Noco. These data suggest that FgChy1 controls hyphal growth, probably by regulating MT assembly and expression in F. graminearum.
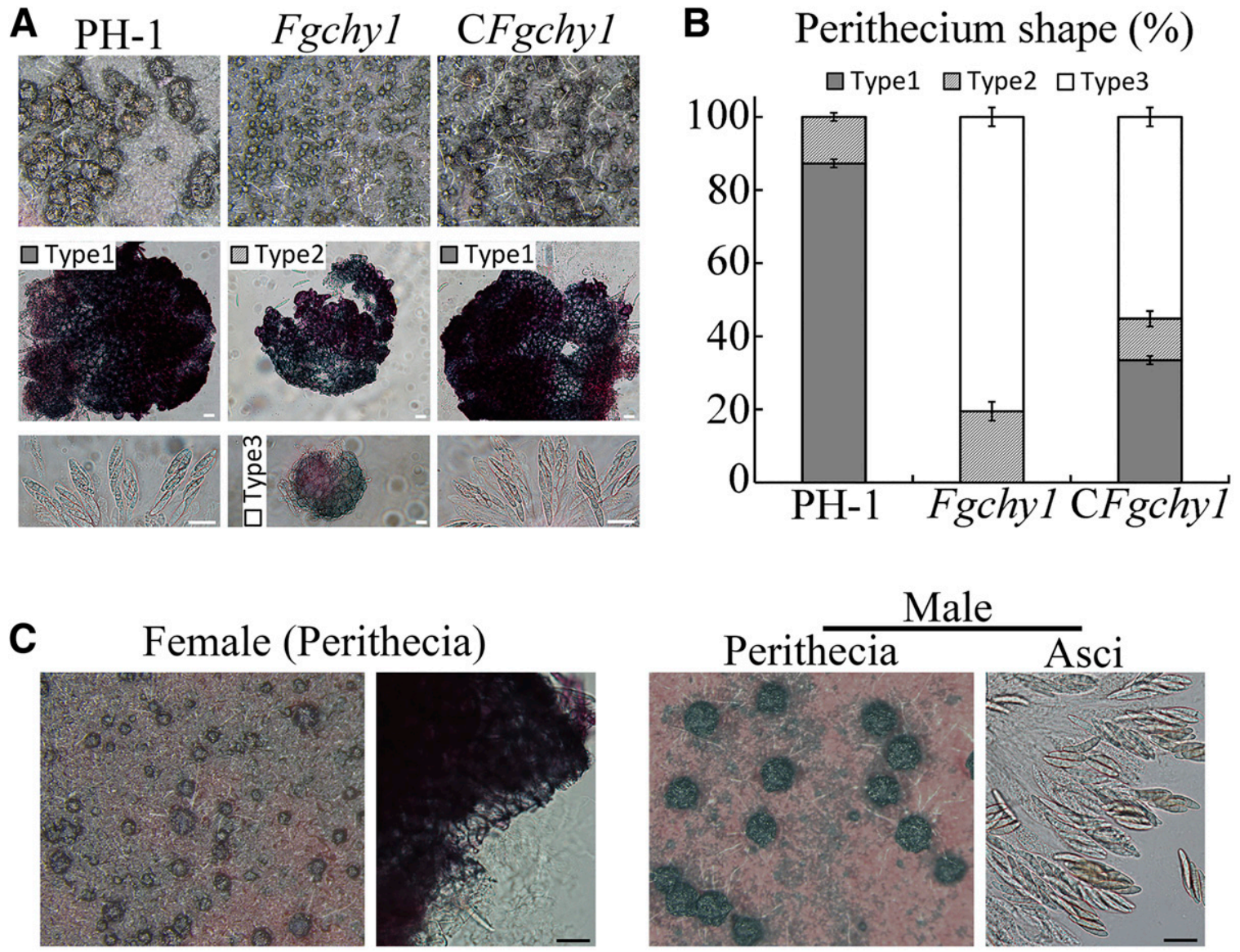

Fig. 5. $\mathrm{FgCHY1}$ is required for female fertility. A, Perithecium (upper and middle) and ascospores (lower) observation in each strain after being cultured on carrot medium for 14 days. Small perithecia (upper, middle, and lower) formed by the Fgchyl mutant. Bar $=20 \mu \mathrm{m}$. B, Statistical analysis for each type (type 1 , normal-sized perithecia; type 2, middle-sized perithecia; type 3, small-sized proto-perithecia) of perithecia. At least 200 perithecia were counted per replicate. C, Two-week-old mating cultures of the Fgchyl mutant used as the female (left) or male (right) crossed with the mat1-1-1 mutant were examined for perithecia and ascospore formation. $\mathrm{Bar}=20 \mu \mathrm{m}$. 
Disassembly of $F$. graminearum MTs by anti-MT drugs affects polarity maintenance and leads to pronounced Fgchy1-style curved hyphae.

MBC and Noco (Sigma-Aldrich) are well-known betatubulin-damaging drugs (Zheng et al. 2016; Zhou et al. 2016). In this study, we observed the growing hyphae of wild type and the Fgchyl mutant in YEPD with (or without) MBC and Noco. As a result, the disassembled $\beta$-tubulin filaments in MBCand Noco-treated PH-1 (Fig. 8F and G) were similar to those in the $\mathrm{FgCHYl}$ deletion mutant. In comparison, the MBC- and Noco-treated Fgchyl mutants showed a seriously inhibited germination rate, more serious $\beta$-tubulin damage, only short fragments of FgTub2, and no FgTub1 GFP fluorescence (Fig. 8H and I). These results indicated that both $\mathrm{MBC}$ and Noco could cause MT damage in $F$. graminearum, and the degree of MT damage in the Fgchyl mutant was more severe than that in the wild type.

After treatment with Noco at $10 \mu \mathrm{M}$ and $\mathrm{MBC}$ at $0.4 \mathrm{ppm}$, $F$. graminearum presented pronounced curved growing hyphae with few septa, which is very similar to the hyphal phenotype of the Fgchyl mutant (Fig. 9A and B). The hyphae of the Fgchyl mutant in YEPD massed into dense clumps, and similar hyphal agglomeration was observed in the PH-1 and CF gchyl strains treated with Noco in YEPD (Supplementary Fig. S3A). Importantly, after anti-MT drug treatment at the same concentration, the growing hyphae of the Fgchyl mutant were more bent than those of the wild type (Fig. 9A), which was consistent with the more severe damage of MT filaments
A

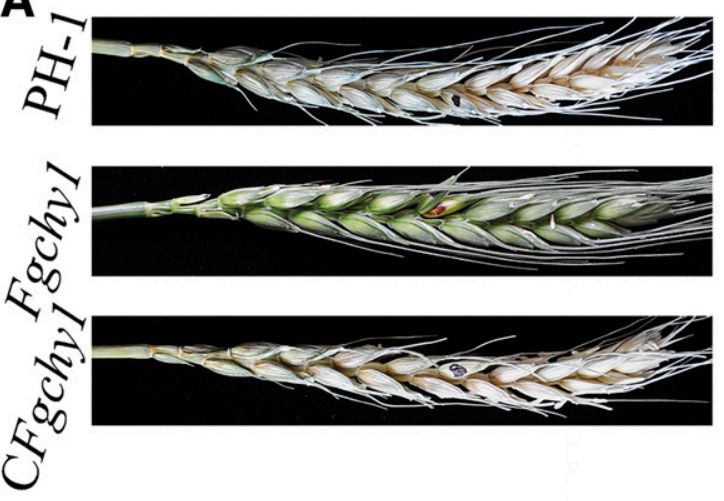

B
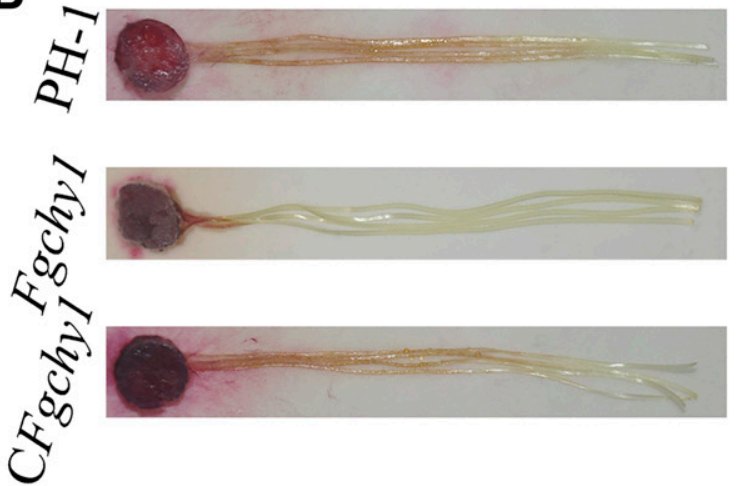

C

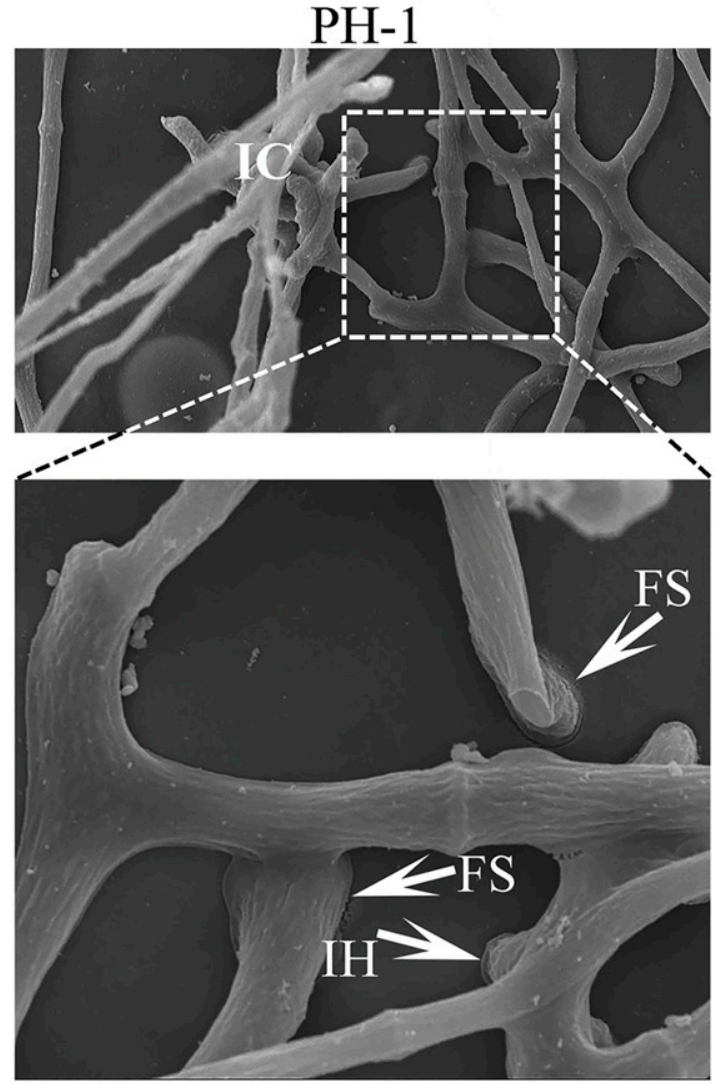

Fgchyl
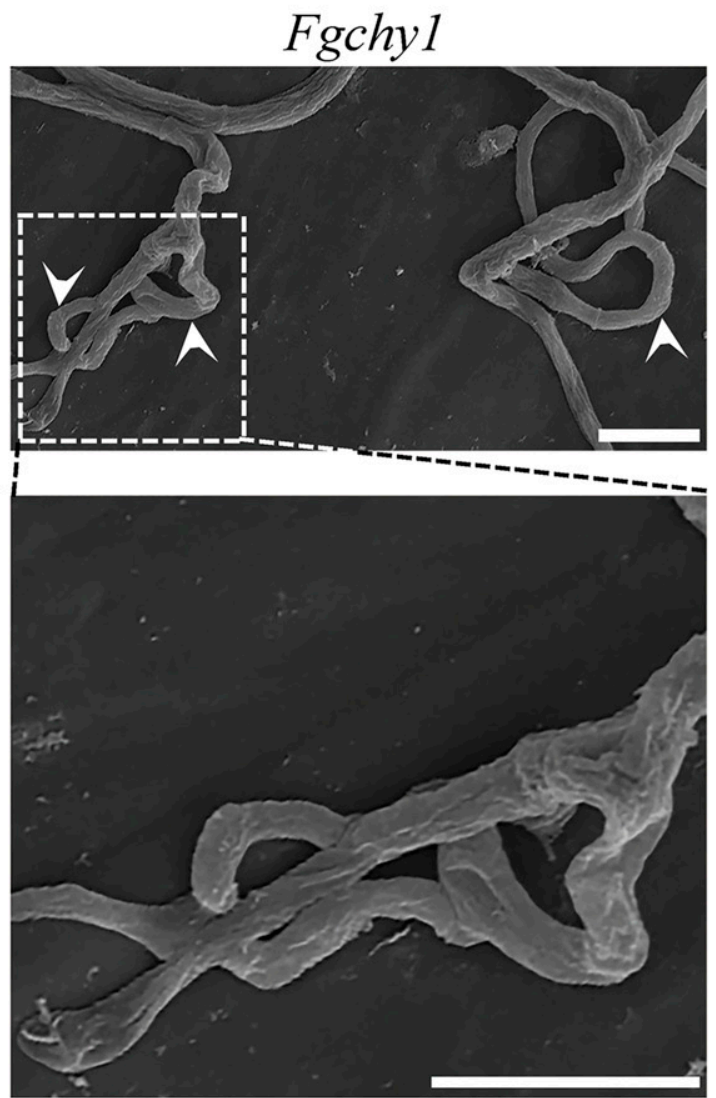

Fig. 6. Infection defects of the Fgchyl mutant in flowering wheat and corn silks. A, Flowering wheat were inoculated with conidia suspension of each strain for 14 days. B, Corn silks were inoculated with hyphal blocks for 5 days. C, Lemmas from the spikelets inoculated with PH-1 and the Fgchy 1 mutant were examined by scanning electron microscopy at $36 \mathrm{~h}$ postinfection. White arrows mark the penetration sites of the inner surface. White arrowheads mark the bending running hyphae. $\mathrm{IC}=$ infection cushion, $\mathrm{FS}=$ foot structure, $\mathrm{IH}=$ infection hypha. Bar $=10 \mu \mathrm{m}$. 
(Fig. 8H and I). These results indicate that beta-tubulin contributes to guiding the growth direction in $F$. graminearum and that the curved hyphae of the Fgchyl mutant were caused by disassembled MTs.

\section{$\mathrm{FgCHY} 1$ is responsible for tolerance to anti-MT drugs.}

The conidia of each strain were inoculated on PDA plates with (or without) different concentrations of MBC and Noco. The inhibition rates of the Fgchyl mutant were up to 57 and $48 \%$, respectively, in PDA with $5 \mu \mathrm{M}$ Noco and $0.1 \mathrm{ppm} \mathrm{MBC}$
$\left(\mathrm{EC}_{50}=0.599 \pm 0.04 \mathrm{ppm}\right)($ Liu et al. 2019). Under the same conditions, WT showed 35 and $6 \%$ inhibition, respectively (Fig. 9C and D). Noco and MBC at concentrations of $20 \mu \mathrm{M}$ and $0.5 \mathrm{ppm}$, respectively, almost completely inhibited the growth of Fgchyl (Fig. 9C and D). In comparison, the inhibition rate in PDA with $0.2 \mathrm{ppm}$ tebuconazole (average $\mathrm{EC}_{50}=0.157$ ppm) (Sun et al. 2014) showed no obvious differences between the Fgchyl mutant and PH-1 (Fig. 9C and D). Therefore, the Fgchyl mutant is more specifically sensitive to MT inhibitors than $\mathrm{PH}-1$.

Table 1. Vegetative growth, conidiation, and virulence of Fusarium graminearum strains ${ }^{\vee}$

\begin{tabular}{|c|c|c|c|c|c|}
\hline \multirow[b]{2}{*}{ Strain } & \multicolumn{2}{|c|}{ Growth ratew } & \multirow[b]{2}{*}{ Conidiation $^{\mathrm{x}}$} & \multirow[b]{2}{*}{ Disease index $^{y}$} & \multirow[b]{2}{*}{$\mathbf{D O N}^{\mathbf{z}}$} \\
\hline & PDA & $5 \times$ YEG & & & \\
\hline PH-1 & $13.4 \pm 0.1 \mathrm{~A}$ & $10.8 \pm 0.2 \mathrm{~A}$ & $1.6 \pm 0.3 \mathrm{~A}$ & $16.6 \pm 1.5 \mathrm{~A}$ & $358.6 \pm 43.3 \mathrm{~A}$ \\
\hline Fgchyl & $3.0 \pm 0.1 \mathrm{~B}$ & $3.0 \pm 0.1 \mathrm{~B}$ & $1.0 \pm 0.1 \mathrm{~B}$ & $1.0 \pm 0.8 \mathrm{~B}$ & $34.9 \pm 24.7 \mathrm{~B}$ \\
\hline $\mathrm{CFgchyl}$ & $12.9 \pm 0.3 \mathrm{~A}$ & $10.8 \pm 0.2 \mathrm{~A}$ & $1.5 \pm 0.3 \mathrm{~A}$ & $15.9 \pm 1.8 \mathrm{~A}$ & $308.5 \pm 56.2 \mathrm{~A}$ \\
\hline \multicolumn{6}{|c|}{$\begin{array}{l}\text { Means and standard deviations were calculated from three independent measurements. The same letter indicates that there was no significant difference. } \\
\text { Different letters mark statistically significant difference }(P \leq 0.05) \text {. }\end{array}$} \\
\hline \multicolumn{6}{|c|}{ w Average daily extension in colony radius on potato dextrose agar (PDA) and $5 \times$ yeast extract glucose (YEG) medium. Measured in millimeters per day. } \\
\hline \multicolumn{6}{|c|}{$\mathrm{x}$ Conidiation in 5-day-old carboxymethylcellulose medium cultures $\left(\times 10^{6}\right.$ per milliliter) } \\
\hline \multicolumn{6}{|c|}{ y Disease index was rated by the number of symptomatic spikelets 14 days after inoculation. } \\
\hline \multicolumn{6}{|c|}{ z Deoxynivalenol (DON) production in infected wheat kernels. Values are parts per million. } \\
\hline
\end{tabular}
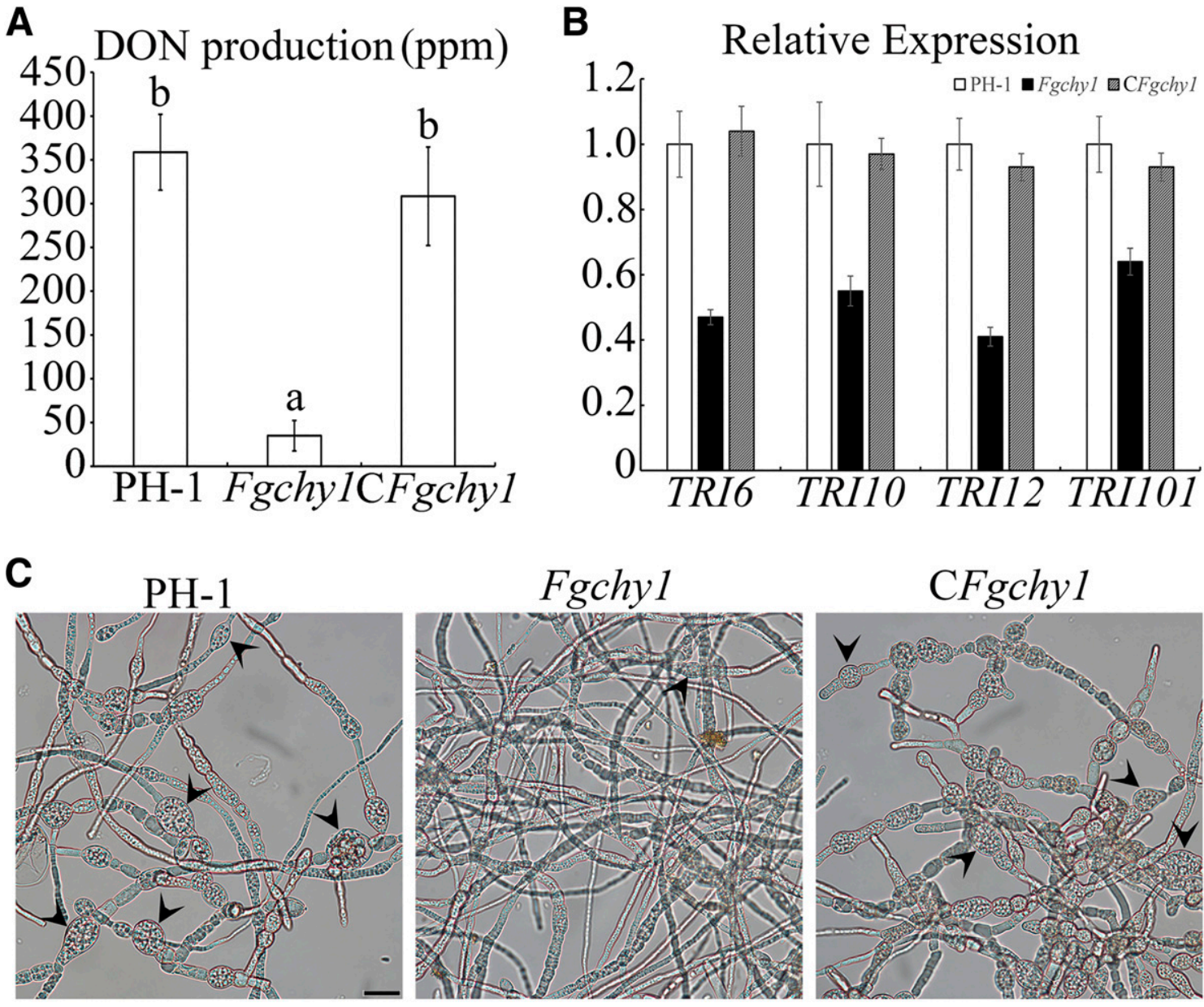

Fig. 7. $F g C H Y 1$ is required for deoxynivalenol (DON) synthesis. A, DON production of the wild-type PH-1, the Fgchyl mutant, and CFgchyl assayed with diseased wheat kernels from symptomatic spikelets. Different letters indicate significant differences. B, Expression levels of the TRI6, TRI10, TRI12, and TRI101 genes in PH-1, the Fgchyl mutant, and CFgchyl. The relative expression level of each gene in PH-1 was arbitrarily set to 1 . The means and standard errors were calculated using data from three independent biological replicates. C, Bulbous examination of each strain in 6-day-old arginine-containing trichothecene biosynthesis induction cultures. Bar $=20 \mu \mathrm{m}$. 


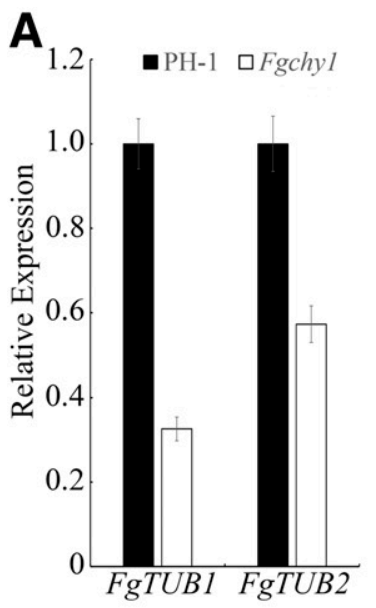

B

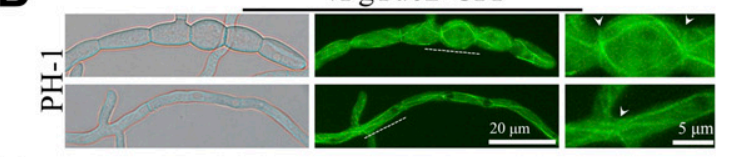

D

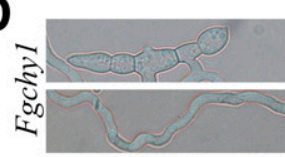

$\mathbf{F}$

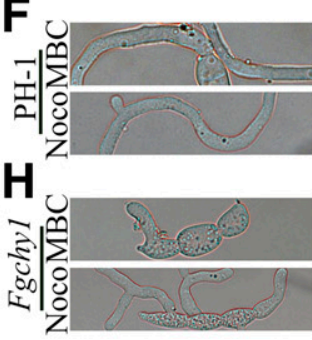

/FgTub2-GFP
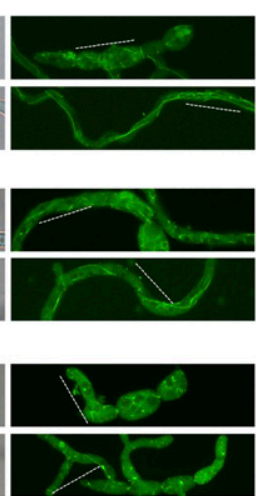
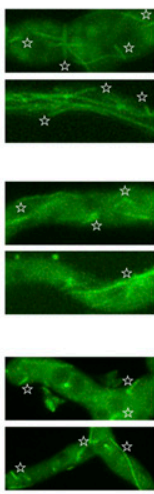

C

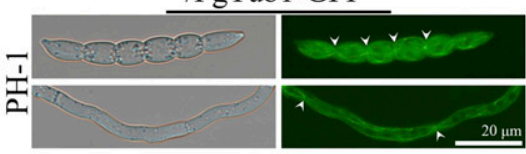

E

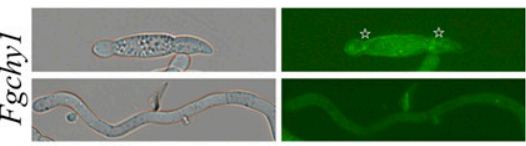

G

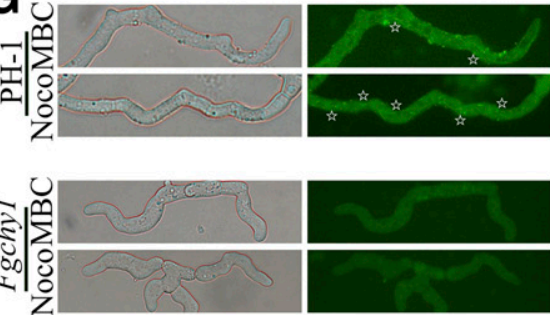

Fig. 8. The expression analysis of beta-tubulin and the fluorescence observation of beta-tubulin filaments. A, The expression level of $F g T U B 1$ and $F g T U B 2$ in the wild-type PH-1 and the Fgchyl mutant. B, Filaments of FgTub2 were organized regularly (marked white arrowheads) in the wild type. C, The normal assembly of FgTub1 filaments (marked white arrowheads) in the wild type. D, The disconnected and messy (marked with stars) FgTub2 filaments in the Fgchy1 mutant. E, Few filaments of FgTub1 in conidia of the Fgchyl mutant were marked with stars and FgTub1 filaments disappeared in hyphae of the Fgchyl mutant. F, PH-1 treated by anti-microtubule agents exhibited disconnected and messy (marked with stars) FgTub2 filaments. G, Few filaments of FgTub1 were marked with stars in hyphae of the $\mathrm{PH}-1$ treated with anti-microtubule agents. $\mathbf{H}$, The Fgchyl mutant treated by anti-microtubule agents exhibited very short pieces of FgTub2 filament (marked with stars). I, The FgTub1-GFP (green fluorescent protein) disappeared in the Fgchyl mutant treated by anti-microtubule agents.

A

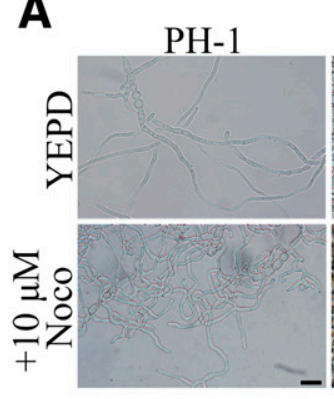

Fgchyl
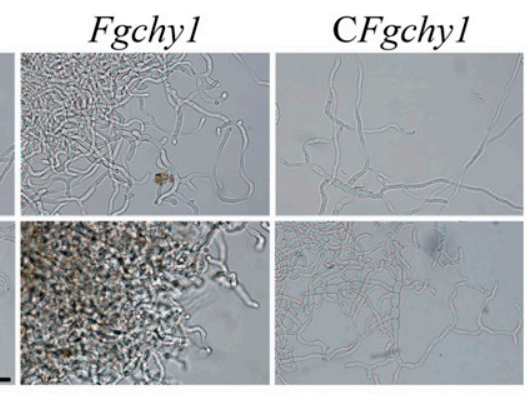

C

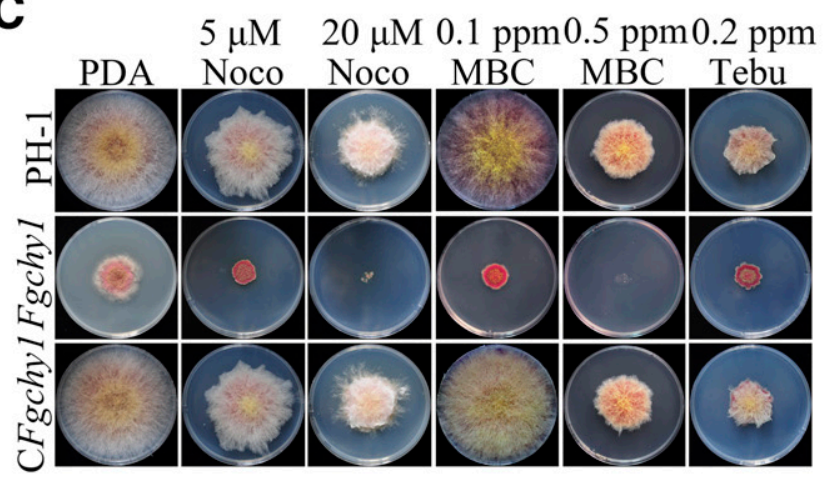

B
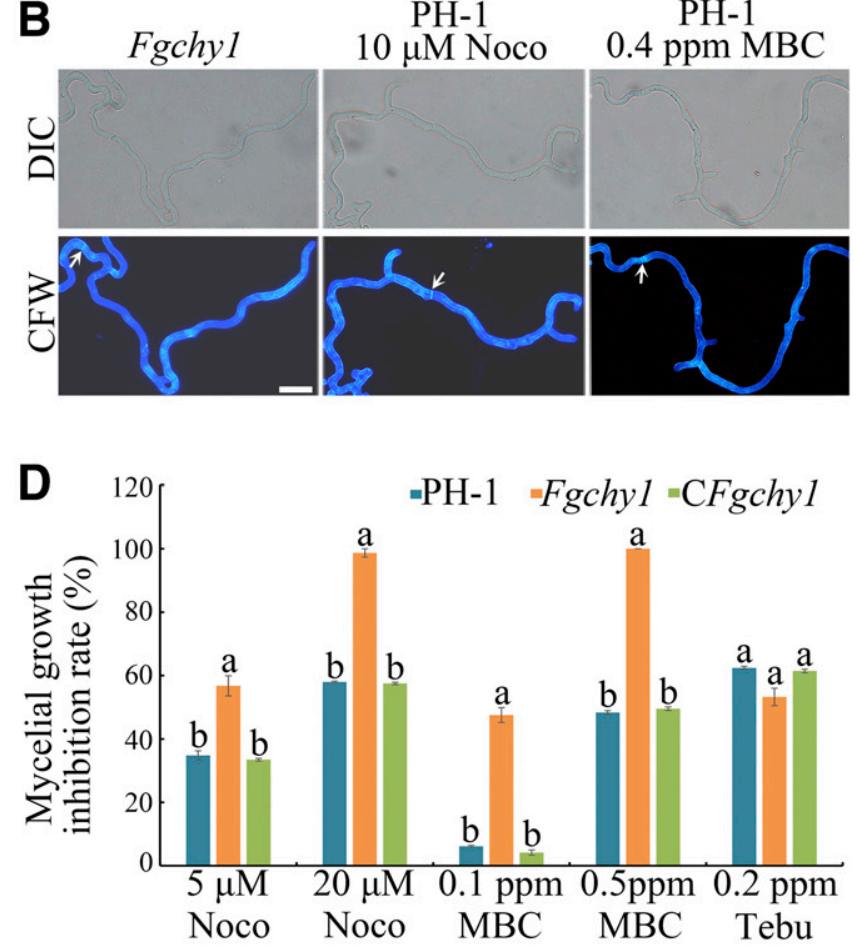

Fig. 9. Anti-microtubule drugs treatment. A, Microscope observation of the growing hyphae of wild-type PH-1, the Fgchyl mutant, and CFgchyl in yeast extract peptone dextrose (YEPD) medium and YEPD containing $10 \mu \mathrm{M}$ Noco (nocodazole). Bar $=20 \mu \mathrm{m}$. B, The growing hyphae of each strain in YEPD with or without microtubule inhibitors $(10 \mu \mathrm{M}$ Noco and $0.4 \mathrm{ppm} \mathrm{MBC}$ [carbendazim]) were stained with CFW (Calcofluor white). White arrows mark the septa. Bar $=20 \mu \mathrm{m}$. C, Each strain was cultured on potato dextrose agar (PDA) plates with 5 and $20 \mu \mathrm{M}$ Noco, 0.1 and 0.5 ppm MBC, and 0.2 ppm tebuconazole (Tebu) for 6 days. D, Statistical analysis of inhibition based on diameters after incubation on PDA medium. Means and standard deviations were calculated from three replicates. Different letters indicate significant difference $(P<0.05)$. 


\section{Deletion of $\mathrm{FgCHY} 1$ influences the location of the actin cortex.}

Considering the interdependent correlation between MTs and actin in hyphal-tip extension (Takeshita et al. 2014), we also examined the localization of actin by expressing the LifeActGFP construct in PH-1 and the Fgchyl mutant. Similar to its localization in wild-type PH-1, LifeAct-GFP was located on the septum and aggregated at the hyphal tip in the mutant (Fig. 10A and B). LifeAct-GFP was arc-shaped and symmetrically distributed at the tip axis in the wild type, whereas in the Fgchyl mutant, LifeAct-GFP gathered into a round spot that was offcenter from the tip axis (Fig. 10A and C). However, the localization of LifeAct-GFP at the septum was not affected in the Fgchyl mutant (Fig. 10B). These results suggest that FgCHYl controls the location of actin in the hyphal tip, but the assembly of F-actin at the septum was independent of FgChy1.

FgCHY1 is required for the localization of FgSpa2.

Spa2p, a polarisome marker, is involved in hyphal apical growth and direction guidance (Araujo-Palomares et al. 2009;
Zheng et al. 2015). In S. cerevisiae, $\mathrm{ScSpa} 2$ is needed for the localization of formin (F-actin polymerization protein) (Köhli et al. 2008). Therefore, we labeled FgSpa2 with GFP and transformed it into the wild type and the Fgchyl mutant. In wild type, FgSpa2-GFP localized near the center of the tip in a dotlike structure. However, in the $F g C H Y 1$ deletion mutant, FgSpa2-GFP often moved to one side of the tip center (Fig. 10D and E). The off-center FgSpa2-GFP appeared to guide a new hyphal growth direction during a 1-min observation (Fig. 10D and E). These results indicate that $F g C H Y 1$ plays an important role in the proper positioning of $\mathrm{FgSpa} 2$, which likely regulates the growth direction and actin localization.

To further investigate the relationship between MTs and FgSpa2 in regulating the unidirectional growth of hyphae, we examined the location of FgSpa2-GFP in PH-1 cells treated with Noco. Consistent with its position in the Fgchyl mutant, FgSpa2 localized on one side of the hyphal-tip axis (Fig. 10F), which further indicates the off-center position of FgSpa2, possibly due to the damaged MTs in the Fgchyl mutant and the Noco-treated wild-type strain.
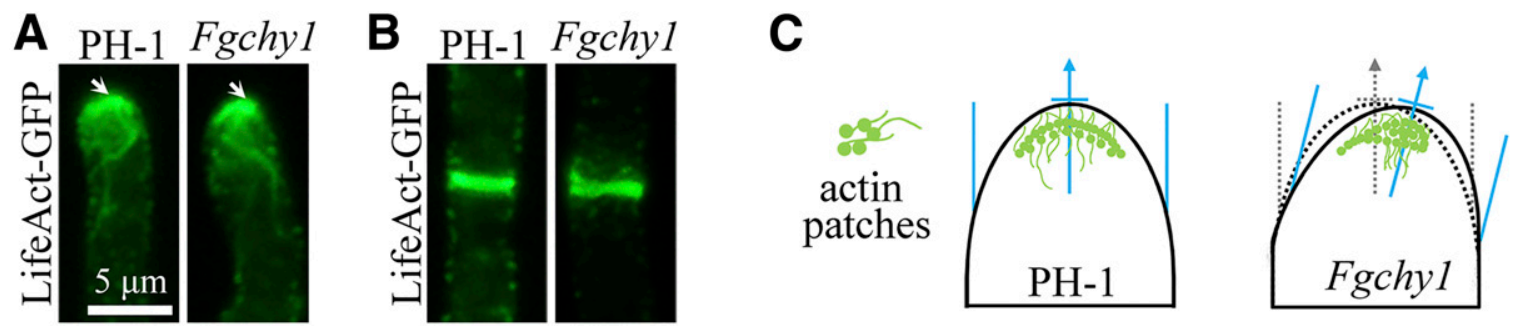

D
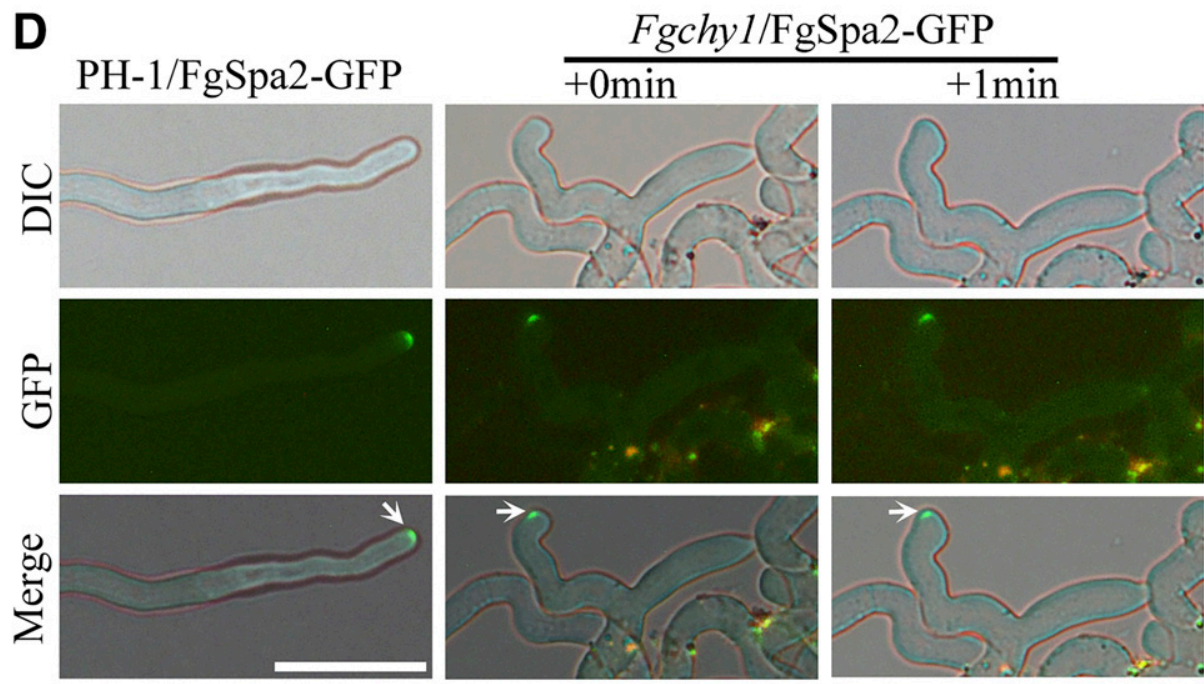

E

$+1 \mathrm{~min}$
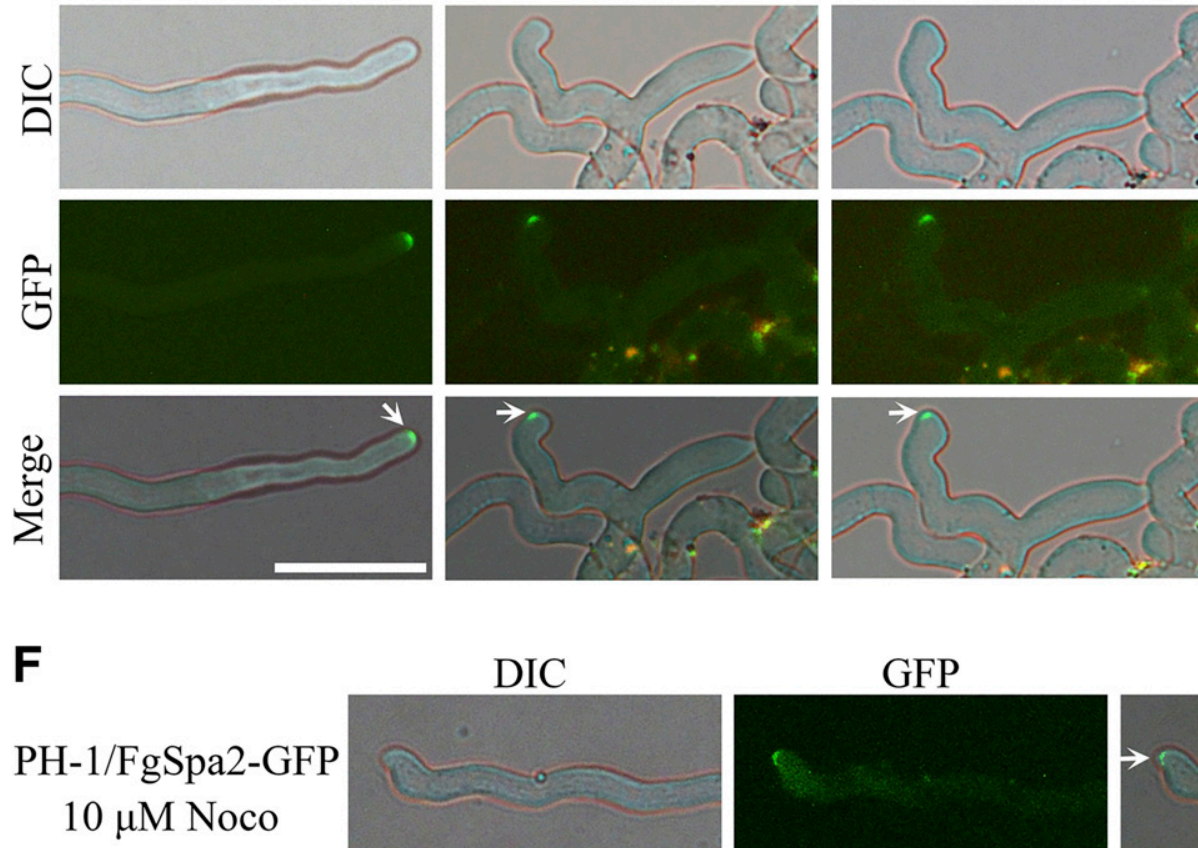

DIC
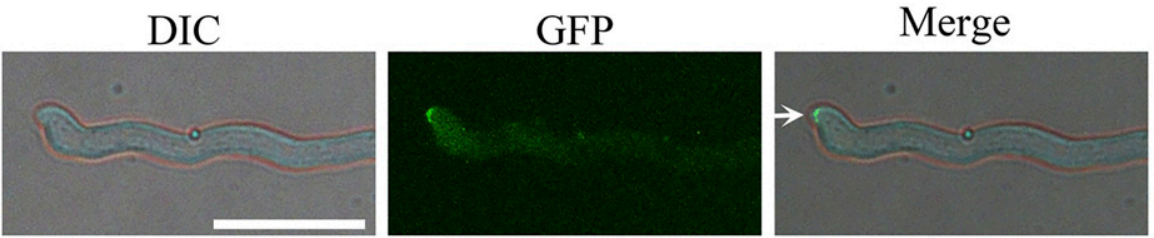

Fig. 10. $\mathrm{FgCHY} 1$ is required for LifeAct cortex and FgSpa2 localization. A, Localization of LifeAct-GFP (green fluorescent protein) in the hyphal-tip regions of PH-1 and the Fgchy1 mutant. White arrows indicate the actin patches in the hyphal tip. B, Localization of LifeAct-GFP in the septum of PH-1 and the Fgchyl mutant. C, Model shows the hyphae tips with actin patches of PH-1 and the Fgchyl mutant. Blue arrows indicate the actual growth axis of hyphae. DIC $=$ differential interference contrast. D, Localization of the FgSpa2-GFP in PH-1 and Fgchy1. FgSpa2 is off-centered in the Fgchy1 mutant, which causes hyphal curved extension in $1 \mathrm{~min}$. The arrows indicate the FgSpa2-GFP protein in the hyphal tips. Bar $=20 \mu \mathrm{m}$. E, Model shows the hyphae tips with FgSpa2 protein of PH-1 and the Fgchyl mutant. Blue arrows indicate the actual growth axis of hyphae along with FgSpa2. F, Localization of the FgSpa2-GFP in PH-1 treated by $10 \mu \mathrm{M}$ Noco (nocodazole). Bar $=20 \mu \mathrm{m}$. 


\section{DISCUSSION}

The Zf-CHY domain is a novel domain that was first reported in filamentous fungi. In this study, three $\mathrm{Zf}-\mathrm{CHY}$ domaincontaining proteins, FgChy1, FgChy2, and FgChy3, were identified in the $F$. graminearum genome. Our findings revealed that FgChy1, FgChy2, and FgChy3 show diversity in their protein structure, evolution, and function. FgChy1 and FgChy2 orthologs are absent in higher plants and animals, and FgChy3 is conserved from lower eukaryotes to higher plants and animals. The deletion of $\mathrm{FgCHY1}$ in $\mathrm{F}$. graminearum results in serious growth defects and reduced pathogenicity, whereas the deletion of $\mathrm{FgCHY} 2$ and $\mathrm{FgCHY} 3$ exerts no effect on growth and pathogenicity. Interestingly, different from the lone $\mathrm{Zf}-$ CHY-containing protein in S. cerevisiae (Supplementary Fig. S1D) or other yeasts in order Saccharomycetales (data not shown), the three corresponding $\mathrm{Zf}-\mathrm{CHY}$-containing proteins are also present in Schizosaccharomyces pombe and even $P$. infestans, and the FgChy1 orthologs in Schizosaccharomyces pombe and $P$. infestans show greater sequence similarity to those of FgChy1 than Hot13 (S. cerevisiae). We suspect that $\mathrm{FgCHY1}$ in $\mathrm{F}$. graminearum and its ortholog in Schizosaccharomyces pombe and even in $P$. infestans might be involved in some shared molecular pathways that are absent in Saccharomycetales.

In this study, we found that FgChy1 exhibits functional differentiation between $F$. graminearum and $S$. cerevisiae. Hot 13 is located in the mitochondrial intermembrane and is dispensable in vegetative growth (Curran et al. 2004); however, FgChy1 is located throughout the whole cell, except the vacuole, and plays an essential role in colony growth in $F$. graminearum. It is also worth mentioning that the molecular mechanism of polarity maintenance regulated by MTs and MT-associated proteins (e.g., TeaA, TeaR, and KipA) is conserved from Schizosaccharomyces pombe to the filamentous fungus $A$. nidulans but shows some differences in S. cerevisiae (Fischer et al. 2008; Takeshita et al. 2013). In this study, we demonstrated that the deletion of $\mathrm{FgCHY} 1$ participates in the regulation of the mycelial direction and is required for polarity maintenance, which might provide a reasonable explanation for a shared molecular pathway of FgCHYl between $F$. graminearum and Schizosaccharomyces pombe (Fischer et al. 2008).

As previously shown in A. nidulans, MTs regulate maintenance of the directionality of tip growth (Taheri-Talesh et al. 2008). To explore how $F g C H Y 1$ modulates the polarized growth of $F$. graminearum, we examined the expression levels of $F g T U B 1$ and $F g T U B 2$ and their morphology in the Fgchyl mutant. As expected, the expression levels of FgTUBl and FgTUB2 in the Fgchyl mutant were decreased compared with those in $\mathrm{PH}-1$, and the deletion of $\mathrm{FgCHY} 1$ resulted in fewer broken and irregular MT filaments, which indicates that FgChy 1 is responsible for polarized growth, probably by regulating MT assembly. This conclusion was further supported by the expanded data obtained in this study. First, we confirmed that anti-MT drug treatment (Noco and MCB) resulted in MT disassembly and led to a pronouncedly curved growth of $F$. graminearum, which was very consistent with the phenotypes of the Fgchyl mutant. Second, under anti-MT drug treatment, the Fgchyl mutant showed more curved hyphae and slower growth and the MT filaments in hyphae of the Fgchyl mutant exhibited more serious damage, which indicated that the curved phenotype of Fgchyl was caused by defects in MT assembly.

Spa2 is a main polarisome component located in the center of the hyphal tip and controls the growth direction and the maximal growth rate in Ashbya gossypii and $F$. graminearum (Knechtle et al. 2003; Zheng et al. 2015). In our study, we found that FgSpa2 is located in an off-center position in the Fgchyl mutant and alters the original growth direction of the hyphae. However, unlike the apical position of $\mathrm{FgSpa} 2$ in growing hyphae, FgChy1-GFP was localized in all cells except vacuoles and did not show enrichment at the top of hyphae (Fig. 1D), which suggests that FgChy1 likely indirectly regulates the position of the tip growth apparatus of FgSpa2 to control the growth direction. Thereafter, FgSpa2 was found in the same off-center location in the MBC-treated wild-type strain (with unstable MTs), which indicated that damaged betatubulin likely resulted in an inaccurate location of FgSpa2, and this finding further suggests that the growth-orientation selection FgChy 1 takes charge of depends on the position of FgSpa2, which is probably directly mediated by MTs in F. graminearum.

Actin and the MT cytoskeleton are closely related during fungal growth (Takeshita et al. 2014). In A. nidulans, TeaA, which is associated with the hyphal growth direction, is delivered by growing MTs to hyphal tips and is involved in SepA recruitment, which is essential for actin polymerization (Higashitsuji et al. 2009). In this study, the location of actin at the cortex of the hyphal tip assembled to one side in the Fgchyl mutant, which might be directly related to its defects in MT assembly. In addition, in $S$. cerevisiae, the polarisome participates in polarization by directing the localized assembly of actin filaments (Köhli et al. 2008; Pruyne and Bretscher 2000). In the present study, FgSpa2 was located in an off-center position in the Fgchyl mutant, which might also explain the position of actin in the hyphal tip of the Fgchyl mutant. In addition, actin-related proteins reportedly regulate the hyphal branch (polarity establishment) (Tang et al. 2018; Upadhyay and Shaw 2008) and there is little evidence showing that actin regulates the growth direction (polarity maintenance). However, in this study, the off-center localization of LifeAct-GFP at hyphal tips was likely involved in the directionless hyphal growth of the Fgchyl mutant, which also suggests that actin patches also play a role in hyphal linear extension. In addition, despite the reduction in septal formation, the localization of actin at the septum was not affected in the Fgchyl mutant. Therefore, FgChy1 regulates septum formation independently of the actin ring.

In asexual and sexual reproduction, the production of conidiophores and perithecia is increased in the $\mathrm{FgCHY}$ deletion mutant, which indicates that $\mathrm{FgCHYl}$ exerts a negative effect on reproduction. Asexual or sexual reproductive structures that produce spores form under nutrient-limited or stressful conditions, although the regulatory mechanism is unclear (Riquelme et al. 2018). Therefore, the increased production of conidiophores and perithecia in the Fgchyl mutant is likely due to unstable extension of the mycelium tip, which influences the utilization of nutrition or materials. The results of this study demonstrate a critical role for $\mathrm{FgCHY1}$ in ascogonia and asci formation and female fertility. In yeast sex, the position and intensity of key polar proteins fluctuate with changes in the mating pheromone concentration. The wandering of polarityrelated proteins might facilitate the reorientation of growth toward pheromones, which are critical for mating (Arkowitz 2013). Therefore, the hyphal growth direction possibly plays an important role in sexual mating. The defects in ascogonia formation observed in the Fgchyl mutant during sexual reproduction are somehow correlated with its defects in the unidirectional growth of $F$. graminearum. In addition, no asci or ascospores were produced in the Fgchyl mutant. This defect is similar to that observed with the Fgtubl mutant at the sexual stage (Zhao et al. 2014). The location of FgTub1 was severely damaged in the Fgchyl mutant, which supports the functions of $\mathrm{FgCHYl}$ in sterile perithecia production. 
In the present study, disruption of $\mathrm{FgCHYl}$ in $\mathrm{F}$. graminearum significantly reduced the pathogenicity. Scab symptoms caused by the Fgchyl mutant were only observed on the inoculated site at $14 \mathrm{dpi}$. One contributing factor to the defects in plant infection could be related to the observed reduction in the growth rate. In addition, in pathogenic filamentous fungi, tip growth plays an important role in host infection (Riquelme et al. 2018). Research on various pathogenic fungi has found that dysfunctions of molecular factors associated with polarized growth (wavy or multibranched hyphae) seriously reduce the pathogenicity or virulence (Gupta et al. 2015; Valinluck et al. 2014). In the present study, the Fgchyl mutant showed defects in infection structure formation and penetration through the lemma epidermis at $36 \mathrm{hpi}$, and only curved hyphae were observed. It is possible that the loss of mycelial polarity in the Fgchyl mutant is responsible for the observed defects in infection structure formation, which account for defects in the induction of symptoms and spreading in infected wheat heads. In addition, DON is an important virulence factor in $F$. graminearum (Lowe et al. 2012). The deletion of $\mathrm{FgCHYl}$ in $\mathrm{F}$. graminearum decreased DON production and DON biosynthesis gene expression, which also contributes to its defects in plant infection. The reduced pathogenicity of the Fgchyl mutant with damaged beta-tubulin is also consistent with the reduced virulence of FgTUB1 and FgTUB2 single-deletion mutants described previously (Wang et al. 2019).

Until now, little has been known about the roles of the $\mathrm{Zf}$ $\mathrm{CHY}$ domain, even though it is ubiquitous in eukaryotes. FgChy1, which was identified in this study, is a Zf-CHY domain-containing protein that plays important roles in MT assembly, polarity maintenance, development, and pathogenicity. In particular, polarized growth is the dominant growth form of filamentous fungi, and the sequence of FgChy1 shows high similarity with that of its homologs among filamentous microorganisms. These findings remind us that the $\mathrm{Zf}-\mathrm{CHY}$ domain likely contributes to polarity maintenance, development, and virulence in pathogenic filamentous fungi. $\mathrm{Zf}-\mathrm{CHY}$ in higher eukaryotes often coexists with other zinc finger structures (e.g., RING) (Cui et al. 2015; Leng et al. 2003). Similarly, the Zf-CHY domains of FgChy2 and FgChy3, which are the other two $\mathrm{Zf}-\mathrm{CHY}$ domain-containing proteins in $F$. graminearum, are coupled with RING fingers. However, the deletion of FgChy 2 and $\mathrm{FgChy} 3$ exerts no effect on $F$. graminearum growth and pathogenicity. Therefore, Zf-CHYs exhibit functional diversity in different protein structures.

The functional role of FgChy1 and its orthologs has barely been studied in fungi. In this study, we demonstrated that the $\mathrm{Zf}-\mathrm{CHY}$ protein $\mathrm{FgCHY}$ plays important roles in the pathogenesis, mycelial unidirectional growth, FgSpa2-GFP localization, and MT assembly in $F$. graminearum. The twisted extension of hyphae is a striking cellular defect in Fgchyl that is related to the fact the off-center tip location of FgSpa2 is likely directly mediated by damaged MTs. The hyphal twisting extent is closely related to $F$. graminearum infection and colonization of host tissue. Our data also clarifies that the damage to MTs resulting from chemical treatment also affects the linear extension of $F$. graminearum, which further supports the notion that MTs play critical roles in unidirectional hyphal growth in filamentous fungi. Characterization of the functions of FgChy1 in $F$. graminearum provides a new understanding of the role of the Zf-CHY domain in regulating MT dynamics and virulence in fungi, particularly pathogenic filamentous fungi. However, proteins acting at MTs and the interaction mechanisms are rather abundant and complex. Additional research is needed to understand the relationship and coordination mechanism among FgChy1, MTs, actin, and the polarisome complex in the unidirectional growth of $F$. graminearum.

\section{MATERIALS AND METHODS}

\section{Strains and culture conditions.}

Wild-type $F$. graminearum PH-1 (Cuomo et al. 2007) and the various transformants used in this study (Supplementary Table S2) were cultured on PDA plates, CM, and carrot agar plates for assays of their growth rate, colony morphology, and sexual reproduction, as previously described (Cavinder et al. 2012). Conidiation in liquid CMC and conidium germination in YEPD broth were performed as described previously (Wang et al. 2011).

\section{Generation of gene-deletion mutants.}

The FgCHY1 gene-knockout mutant was generated using the split-marker approach. The 1.0-kb upstream and 1.0-kb downstream flanking sequences of $\mathrm{FgCHY1}$ were amplified and fused with hygromycin phosphotransferase fragments by overlapping PCR as previously described (Catlett et al. 2003). The resulting PCR products were transformed into protoplasts of the wild type. Hygromycin-resistant transformants were screened by PCR with four pairs of primers (Supplementary Table S1). The same split-marker approach was used to generate the $\mathrm{FgCHY} 2$ and $\mathrm{FgCHY} 3$ deletion mutants. All the primers used in this study are listed in Supplementary Table S1. At least two positive transformants for every gene were used for phenotypic analyses.

\section{Gene complementation and plasmid constructs.}

For the complementation assays, the $\mathrm{FgCHY1-GFP}$ vector was constructed by gap repair. The $\mathrm{FgCHYl}$ open reading frame plus its native promoter were amplified and cotransformed with the XhoI-digested pFL2 plasmid into yeast strain XK1-25 (Bruno et al. 2004) as previously described (Zhou et al. 2011). Transformants expressing $\mathrm{FgCHYl-GFP}$ were analyzed by PCR and GFP signal examination. The same method was used to construct the $F g C H Y 1$-Flag vector with the XhoI-digested pFL7 plasmid, and the resulting transformants were examined by PCR and Western blotting and were named Fgchyl/FgCHY1-Flag. FgTub1-GFP (Wang et al. 2019), FgTub2-GFP (Wang et al. 2019), LifeAct-GFP (pXY198) (Zhou et al. 2012), Histone-GFP (Zhou et al. 2011), and pKNT (Zheng et al. 2016) plasmids were obtained from G. Wang of NWAFU-PU Joint Research Center, College of Plant Protection, Northwest A\&F University, Yangling, China. FgSPA2 was cloned into pKNT using a ClonExpress II one step cloning kit (Vazyme).

\section{Staining and microscopy.}

The PH-1, Fgchyl mutant, PH-1/H1-GFP, and Fgchyl/H1GFP strains were stained with $10 \mu \mathrm{g}$ of CFW per milliliter for $30 \mathrm{~s}$, and the cell walls, septa, and nuclei of the conidia were observed under a Nikon E400 (Li et al. 2018). Nuclei of the complementary strain $\mathrm{CFg}$ chyl with GFP signals were stained with $20 \mathrm{ng}$ of DAPI per milliter for $10 \mathrm{~min}$ (Wang et al. 2011). For vacuole observation, the hyphae of the $\mathrm{CFg}$ chyl strain were stained with CMAC (7-amino-4-chloromethylcoumarin, Keygen Biotech) at a final concentration of $10 \mu \mathrm{M}$ for $15 \mathrm{~min}$ as described ( $\mathrm{Li}$ et al. 2019). Microscopic examination of the conidia, hyphae, and fluorescence signals was performed with a Nikon E400 in this study.

\section{Analysis of the sensitivity of MT inhibitors.}

We inoculated a $10-\mu l$ conidia suspension of each strain onto PDA plates, with (and without) the MT inhibitors MBC and Noco, cultured at $25^{\circ} \mathrm{C}$ for 6 days (Zheng et al. 2016). Conidia of each strain were inoculated into liquid YEPD medium for 15 h, with (and without) Noco, to examine the effect of the inhibitors on conidia germination. 


\section{Infection and DON production assays.}

For the plant infection assays, corn silks at the anthesis stage were inoculated with the hyphal block of each strain as previously described (Cao et al. 2016), and photographs were taken at $5 \mathrm{dpi}$. Infection assays using flowering wheat heads of cultivar Annong 8455 were performed as previously described (Li et al. 2019). The inoculated wheat heads were examined at $14 \mathrm{dpi}$, and the disease index was estimated. Inoculated wheat kernels were collected at $14 \mathrm{dpi}$ for the quantification of mycotoxin production. DON was extracted and purified as previously described (Bluhm et al. 2007). The amount of DON (per milligram of sample) in each sample was determined using a Waters 1525 HPLC system (Shimadzu Corporation).

\section{SEM observations.}

The samples were prepared for SEM according to previous reports (Cao et al. 2016). In brief, lemmas were collected from inoculated spikelets at 36 and $60 \mathrm{hpi}$, were fixed with $2.5 \%$ ( vol $/ \mathrm{vol}$ ) glutaraldehyde overnight at $4{ }^{\circ} \mathrm{C}$, and were then rinsed five times. The samples were dehydrated through an acetone series $(30,50,70,80,90$, and $100 \%$ [vol/vol] $)$ and were then dried with a critical point drier. The processed samples were subsequently observed with an EVO-LS10 scanning microscope (Carl Zeiss) at $10.00 \mathrm{kV}$. At least three independent biological replicates of the wild-type and Fgchyl mutant strains were examined.

\section{qRT-PCR analyses.}

RNA was isolated using the TRIzol reagent (Invitrogen) and were used for cDNA synthesis with the PrimeScript RT reagent kit (TaKaRa), in accordance with manufacturer instructions. The primers used for qRT-PCR analysis are listed in Supplementary Table S1. The actin gene (FGSG_07335) was used as an internal control. The $2^{-\Delta \Delta C T}$ method was used to determine the relative gene expression levels (Livak and Schmittgen 2001). The mean expression level of each gene and the corresponding standard deviation were calculated from three biological replicates.

\section{ACKNOWLEDGMENTS}

We thank X. Zhang for assistance with microtubule inhibitors sensitivity assays. We also thank J.-R. Xu at Purdue University and C. Jiang at Northwest A\&F University for proving the pFL2, pFL7, and pCB1003 vectors and yeast strains.

\section{AUTHOR-RECOMMENDED INTERNET RESOURCE}

EnsemblFungi $F$. graminearum genome database: http://fungi.ensembl.org/Fusarium_graminearum/Info/Index

\section{LITERATURE CITED}

Alexander, N. J., Proctor, R. H., and McCormick, S. P. 2009. Genes, gene clusters, and biosynthesis of trichothecenes and fumonisins in Fusarium. Toxin Rev. 28:198-215.

Araujo-Palomares, C. L., Riquelme, M., and Castro-Longoria, E. 2009. The polarisome component SPA-2 localizes at the apex of Neurospora crassa and partially colocalizes with the Spitzenkörper. Fungal Genet. Biol. 46: 551-563.

Arkowitz, R. A. 2013. Cell polarity: Wanderful exploration in yeast sex. Curr. Biol. 23:R10-R12.

Audenaert, K., Callewaert, E., Höfte, M., De Saeger, S., and Haesaert, G. 2010. Hydrogen peroxide induced by the fungicide prothioconazole triggers deoxynivalenol (DON) production by Fusarium graminearum. BMC Microbiol. 10:112.

Bluhm, B. H., Zhao, X., Flaherty, J. E., Xu, J. R., and Dunkle, L. D. 2007. RAS2 regulates growth and pathogenesis in Fusarium graminearum. Mol. Plant-Microbe Interact 20:627-636.
Bruno, M., Flaus, A., and Owen-Hughes, T. 2004. Site-specific attachment of reporter compounds to recombinant histones. Methods Enzymol. 375: 211-228.

Cao, S., Zhang, S., Hao, C., Liu, H., Xu, J. R., and Jin, Q. 2016. FgSsn3 kinase, a component of the mediator complex, is important for sexual reproduction and pathogenesis in Fusarium graminearum. Sci. Rep. 6: 22333.

Catlett, N., Lee, B. N., Yoder, O., and Turgeon, G. 2003. Split-marker recombination for efficient targeted deletion of fungal genes. Fungal Genet. Newsl. 50:9-11.

Cavinder, B., Sikhakolli, U., Fellows, K. M., and Trail, F. 2012. Sexual development and ascospore discharge in Fusarium graminearum. J. Vis. Exp. 61:e3895.

Chu, Z., Chen, J., Nyporko, A., Han, H., Yu, Q., and Powles, S. 2018. Novel $\alpha$-tubulin mutations conferring resistance to dinitroaniline herbicides in Lolium rigidum. Front. Plant Sci. 9:97.

Cui, L. G., Shan, J. X., Shi, M., Gao, J. P., and Lin, H. X. 2015. DCA1 acts as a transcriptional co-activator of DST and contributes to drought and salt tolerance in rice. PLoS Genet. 11:e1005617.

Cuomo, C. A., Güldener, U., Xu, J. R., Trail, F., Turgeon, B. G., Di Pietro, A., Walton, J. D., Ma, L. J., Baker, S. E., Rep, M., Adam, G., Antoniw, J., Baldwin, T., Calvo, S., Chang, Y. L., Decaprio, D., Gale, L. R., Gnerre, S., Goswami, R. S., Hammond-Kosack, K., Harris, L. J., Hilburn, K., Kennell, J. C., Kroken, S., Magnuson, J. K., Mannhaupt, G., Mauceli, E., Mewes, H. W., Mitterbauer, R., Muehlbauer, G., Münsterkötter, M., Nelson, D., O’donnell, K., Ouellet, T., Qi, W., Quesneville, H., Roncero, M. I., Seong, K. Y., Tetko, I. V., Urban, M., Waalwijk, C., Ward, T. J., Yao, J., Birren, B. W., and Kistler, H. C. 2007. The Fusarium graminearum genome reveals a link between localized polymorphism and pathogen specialization. Science 317:1400-1402.

Curran, S. P., Leuenberger, D., Leverich, E. P., Hwang, D. K., Beverly, K. N., and Koehler, C. M. 2004. The role of Hot13p and redox chemistry in the mitochondrial TIM22 import pathway. J. Biol. Chem. 279: 43744-43751.

Fischer, R., Zekert, N., and Takeshita, N. 2008. Polarized growth in fungi-Interplay between the cytoskeleton, positional markers and membrane domains. Mol. Microbiol. 68:813-826.

Goswami, R. S., and Kistler, H. C. 2004. Heading for disaster: Fusarium graminearum on cereal crops. Mol. Plant Pathol. 5:515-525.

Gupta, Y. K., Dagdas, Y. F., Martinez-Rocha, A. L., Kershaw, M. J., Littlejohn, G. R., Ryder, L. S., Sklenar, J., Menke, F., and Talbot, N. J. 2015. Septin-dependent assembly of the exocyst is essential for plant infection by Magnaporthe oryzae. Plant Cell 27:3277-3289.

Higashitsuji, Y., Herrero, S., Takeshita, N., and Fischer, R. 2009. The cell end marker protein TeaC is involved in growth directionality and septation in Aspergillus nidulans. Eukaryot. Cell 8:957-967.

Horio, T., and Oakley, B. R. 2005. The role of microtubules in rapid hyphal tip growth of Aspergillus nidulans. Mol. Biol. Cell 16:918-926.

Hwang, J. E., Hong, J. Y., Kim, K., Kim, S. H., Choi, W. Y., Kim, M. J., Sung-Hoon Jung, S. H., Shim, H. J., Bae, W. K., Hwang, E. C., Lee, K. H., Lee, J. H., Cho, S. H., and Chung, I. J. 2013. Class III-tubulin is a predictive marker for taxane-based chemotherapy in recurrent and metastatic gastric cancer. BMC Cancer 13:431.

Jiang, C., Zhang, C., Wu, C., Sun, P., Hou, R., Liu, H., Wang, C., and Xu, J. R. 2016. TRI6 and TRI10 play different roles in the regulation of deoxynivalenol (DON) production by cAMP signalling in Fusarium graminearum. Environ. Microbiol. 18:3689-3701.

Kiso, T., Fujita, K., Ping, X., Tanaka, T., and Taniguchi, M. 2004. Screening for microtubule-disrupting antifungal agents by using a mitotic-arrest mutant of Aspergillus nidulans and novel action of phenylalanine derivatives accompanying tubulin loss. Antimicrob. Agents Chemother. 48:1739-1748.

Knechtle, P., Dietrich, F., and Philippsen, P. 2003. Maximal polar growth potential depends on the polarisome component AgSpa2 in the filamentous fungus Ashbya gossypii. Mol. Biol. Cell 14:4140-4154.

Köhli, M., Galati, V., Boudier, K., Roberson, R. W., and Philippsen, P. 2008. Growth-speed-correlated localization of exocyst and polarisome components in growth zones of Ashbya gossypii hyphal tips. J. Cell Sci. 121: 3878-3889.

Leng, R. P., Lin, Y., Ma, W., Wu, H., Lemmers, B., Chung, S., Parant, J. M., Lozano, G., Hakem, R., and Benchimol, S. 2003. Pirh2, a p53-induced ubiquitin-protein ligase, promotes p53 degradation. Cell 112:779-791.

Li, B., Dong, X., Zhao, R., Kou, R., Zheng, X., and Zhang, H. 2019. The t-SNARE protein FgPep12, associated with FgVam7, is essential for ascospore discharge and plant infection by trafficking $\mathrm{Ca}^{2+}$ ATPase FgNeo1 between Golgi and endosome/vacuole in Fusarium graminearum. PLoS Pathog. 15:e1007754. 
Li, C., Cao, S., Zhang, C., Zhang, Y., Zhang, Q., Xu, J. R., and Wang, C. 2018. MoCDC14 is important for septation during conidiation and appressorium formation in Magnaporthe oryzae. Mol. Plant Pathol. 19: 328-340.

Liu, N., Wu, S., Dawood, D. H., Tang, G., Zhang, C., Liang, J., Chen, Y., and Ma, Z. 2019. The b-ZIP transcription factor FgTfmI is required for the fungicide phenamacril tolerance and pathogenecity in Fusarium graminearum. Pest Manag. Sci. 75:3312-3322.

Liu, X., Yin, Y., Wu, J., Jiang, J., and Ma, Z. 2010. Identification and characterization of carbendazim-resistant isolates of Gibberella zeae. Plant Dis. 94:1137-1142.

Liu, Z., Wu, S., Chen, Y., Han, X., Gu, Q., Yin, Y., and Ma, Z. 2017. The microtubule end-binding protein FgEB1 regulates polar growth and fungicide sensitivity via different interactors in Fusarium graminearum. Environ. Microbiol. 19:1791-1807.

Livak, K. J., and Schmittgen, T. D. 2001. Analysis of relative gene expression data using real-time quantitative PCR and the $2^{-\Delta \Delta C(T)}$ method. Methods 25:402-408.

Lowe, R., Jubault, M., Canning, G., Urban, M., and Hammond-Kosack, K. E. 2012. The induction of mycotoxins by trichothecene producing Fusarium species. Methods Mol. Biol. 835:439-455.

Ojima, I., Kumar, K., Awasthi, D., and Vineberg, J. G. 2014. Drug discovery targeting cell division proteins, microtubules and FtsZ. Bioorg. Med. Chem. 22:5060-5077.

Pruyne, D., and Bretscher, A. 2000. Polarization of cell growth in yeast. I. Establishment and maintenance of polarity states. J. Cell Sci. 113:365-375.

Qiu, J., Xu, J., Yu, J., Bi, C., Chen, C., and Zhou, M. 2011. Localisation of the benzimidazole fungicide binding site of Gibberella zeae $\beta 2$-tubulin studied by site-directed mutagenesis. Pest Manag. Sci. 67:191-198.

Riquelme, M. 2013. Tip growth in filamentous fungi: A road trip to the apex. Annu. Rev. Microbiol. 67:587-609.

Riquelme, M., Aguirre, J., Bartnicki-García, S., Braus, G. H., Feldbrügge, M., Fleig, U., Hansberg, W., Herrera-Estrella, A., Kämper, J., Kück, U., Mouriño-Pérez, R. R., Takeshita, N., and Fischer, R. 2018. Fungal morphogenesis, from the polarized growth of hyphae to complex reproduction and infection structures. Microbiol. Mol. Biol. Rev. 82:e00068-17.

Seong, K. Y., Pasquali, M., Zhou, X., Song, J., Hilburn, K., McCormick, S., Dong, Y., Xu, J. R., and Kistler, H. C. 2009. Global gene regulation by Fusarium transcription factors Tri6 and Tri10 reveals adaptations for toxin biosynthesis. Mol. Microbiol. 72:354-367.

Spolti, P., Del Ponte, E. M., Dong, Y., Cummings, J. A., and Bergstrom, G. C. 2014. Triazole sensitivity in a contemporary population of Fusarium graminearum from New York wheat and competitiveness of a tebuconazole-resistant isolate. Plant Dis. 98:607-613.

Starkey, D. E., Ward, T. J., Aoki, T., Gale, L. R., Kistler, H. C., Geiser, D. M., Suga, H., Tóth, B., Varga, J., and O’Donnell, K. 2007. Global molecular surveillance reveals novel Fusarium head blight species and trichothecene toxin diversity. Fungal Genet. Biol. 44:1191-1204.

Sun, H. Y., Zhu, Y. F., Liu, Y. Y., Deng, Y. Y., Li, W., Zhang, A. X., and Chen, H. G. 2014. Evaluation of tebuconazole for the management of Fusarium head blight in China. Australas. Plant Pathol. 43:631-638.

Taheri-Talesh, N., Horio, T., Araujo-Bazán, L., Dou, X., Espeso, E. A., Peñalva, M. A., Osmani, S. A., and Oakley, B. R. 2008. The tip growth apparatus of Aspergillus nidulans. Mol. Biol. Cell 19:1439-1449.

Takeshita, N., Manck, R., Grün, N., de Vega, S. H., and Fischer, R. 2014. Interdependence of the actin and the microtubule cytoskeleton during fungal growth. Curr. Opin. Microbiol. 20:34-41.

Takeshita, N., Mania, D., Herrero, S., Ishitsuka, Y., Nienhaus, G. U., Podolski, M., Howard, J., and Fischer, R. 2013. The cell-end marker
TeaA and the microtubule polymerase AlpA contribute to microtubule guidance at the hyphal tip cortex of Aspergillus nidulans to provide polarity maintenance. J. Cell Sci. 126:5400-5411.

Tang, W., Gao, C., Wang, J., Yin, Z., Zhang, J., Ji, J., Zhang, H., Zheng, X., Zhang, Z., and Wang, P. 2018. Disruption of actin motor function due to MoMyo5 mutation impairs host penetration and pathogenicity in Magnaporthe oryzae. Mol. Plant Pathol. 19:689-699.

Upadhyay, S., and Shaw, B. D. 2008. The role of actin, fimbrin and endocytosis in growth of hyphae in Aspergillus nidulans. Mol. Microbiol. 68:690-705.

Valinluck, M., Woraratanadharm, T., Lu, C. Y., Quintanilla, R. H., Jr., and Banuett, F. 2014. The cell end marker Tea4 regulates morphogenesis and pathogenicity in the basidiomycete fungus Ustilago maydis. Fungal Genet. Biol. 66:54-68.

Walter, S., Nicholson, P., and Doohan, F. M. 2010. Action and reaction of host and pathogen during Fusarium head blight disease. New Phytol. 185:54-66.

Wang, C., Zhang, S., Hou, R., Zhao, Z., Zheng, Q., Xu, Q., Zheng, D., Wang, G., Liu, H., Gao, X., Ma, J. W., Kistler, H. C., Kang, Z., and Xu, J. R. 2011. Functional analysis of the kinome of the wheat scab fungus Fusarium graminearum. PLoS Pathog. 7:e1002460.

Wang, H., Chen, D., Li, C., Tian, N., Zhang, J., Xu, J. R., and Wang, C. 2019. Stage-specific functional relationships between Tub1 and Tub2 beta-tubulins in the wheat scab fungus Fusarium graminearum. Fungal Genet. Biol. 132:103251.

Yeh, E., Yang, C., Chin, E., Maddox, P., Salmon, E. D., Lew, D. J., and Bloom, K. 2000. Dynamic positioning of mitotic spindles in yeast: Role of microtubule motors and cortical determinants. Mol. Biol. Cell 11: 3949-3961.

Zeng, C. J., Kim, H. R., Vargas Arispuro, I., Kim, J. M., Huang, A. C., and Liu, B. 2014. Microtubule plus end-tracking proteins play critical roles in directional growth of hyphae by regulating the dynamics of cytoplasmic microtubules in Aspergillus nidulans. Mol. Microbiol. 94: 506-521.

Zhang, Y. J., Yu, J. J., Zhang, Y. N., Zhang, X., Cheng, C. J., Wang, J. X., Hollomon, D. W., Fan, P. S., and Zhou, M. G. 2009. Effect of carbendazim resistance on trichothecene production and aggressiveness of Fusarium graminearum. Mol. Plant-Microbe Interact 22:1143-1150.

Zhao, Z., Liu, H., Luo, Y., Zhou, S., An, L., Wang, C., Jin, Q., Zhou, M., and $\mathrm{Xu}$, J. R. 2014. Molecular evolution and functional divergence of tubulin superfamily in the fungal tree of life. Sci. Rep. 4:6746.

Zheng, H., Zheng, W., Wu, C., Yang, J., Xi, Y., Xie, Q., Zhao, X., Deng, X., Lu, G., Li, G., Ebbole, D., Zhou, J., and Wang, Z. 2015. Rab GTPases are essential for membrane trafficking-dependent growth and pathogenicity in Fusarium graminearum. Environ. Microbiol. 17:4580-4599.

Zheng, W., Zheng, H., Zhao, X., Zhang, Y., Xie, Q., Lin, X., Chen, A., Yu, W., Lu, G., Shim, W. B., Zhou, J., and Wang, Z. 2016. Retrograde trafficking from the endosome to the trans-Golgi network mediated by the retromer is required for fungal development and pathogenicity in Fusarium graminearum. New Phytol. 210:1327-1343.

Zhou, X., Li, G., and Xu, J. R. 2011. Efficient approaches for generating GFP fusion and epitope-tagging constructs in filamentous fungi. Methods Mol. Biol. 722:199-212.

Zhou, X., Zhang, H., Li, G., Shaw, B., and Xu, J. R. 2012. The cyclaseassociated protein Cap1 is important for proper regulation of infectionrelated morphogenesis in Magnaporthe oryzae. PLoS Pathog. 8:e1002911.

Zhou, Y., Zhu, Y., Li, Y., Duan, Y., Zhang, R., and Zhou, M. 2016. $\beta 1$ tubulin rather than $\beta 2$ tubulin is the preferred binding target for carbendazim in Fusarium graminearum. Phytopathology 106:978-985. 